\title{
ON TARSKI'S AXIOMATIC FOUNDATIONS OF THE CALCULUS OF RELATIONS
}

\author{
HAJNAL ANDRÉKA, STEVEN GIVANT, PETER JIPSEN, AND ISTVÁN NÉMETI
}

\begin{abstract}
It is shown that Tarski's set of ten axioms for the calculus of relations is independent in the sense that no axiom can be derived from the remaining axioms. It is also shown that by modifying one of Tarski's axioms slightly, and in fact by replacing the right-hand distributive law for relative multiplication with its left-hand version, we arrive at an equivalent set of axioms which is redundant in the sense that one of the axioms, namely the second involution law, is derivable from the other axioms. The set of remaining axioms is independent. Finally, it is shown that if both the left-hand and right-hand distributive laws for relative multiplication are included in the set of axioms, then two of Tarski's other axioms become redundant, namely the second involution law and the distributive law for converse. The set of remaining axioms is independent and equivalent to Tarski's axiom system.
\end{abstract}

\section{INTRODUCTION}

In a series of publications over a period of 40 years, Augustus De Morgan [2], Charles Sanders Peirce (see, in particular, 22]) and Ernst Schröder 24 developed a calculus of binary relations that extended Boole's calculus of classes. At the time it was considered one of the cornerstones of mathematical logic, and indeed, in 1903 Bertrand Russell [23] wrote:

The subject of symbolic logic is formed by three parts: the calculus of propositions, the calculus of classes, and the calculus of relations.

The celebrated theorem of Löwenheim [11] (which today would be formulated as stating that every formula valid in some model must in fact be valid in some countable model) was proved in the framework of the calculus of relations.

Interest in the theory gradually dwindled after Löwenheim's paper, until 1941, when Alfred Tarski [25] introduced an abstract algebraic axiomatization of the calculus of relations, announced several very deep results that he had obtained concerning the theory, and raised a number of problems that stimulated interest and research in the subject for decades to come. Tarski's original axiomatization did not consist entirely of equations, but he indicated that such an equational axiomatization was possible (see pp. 86-87 of [25]), and by 1943 he had worked out such an axiomatization (see [26]). This equational axiomatization, with minor variations, has subsequently been used by almost all researchers in the field; see, for example, Chin-Tarski [1, Hirsch-Hodkinson [4], Jónsson [5], 6], Maddux [18, McKenzie [20], Monk 21], and Tarski-Givant [27], to name but a few. The models of this set of axioms are called relation algebras.

1991 Mathematics Subject Classification. 03G15, 03B30, 03C05, 03C13. 
In the early 1940s, J. C. C. McKinsey showed that one of Tarski's axioms, the associative law for relative multiplication, is independent of the remaining axioms of the theory. This result was not published at the time, but Tarski preserved McKinsey's independence model by presenting it in an appendix to an unpublished monograph [26], written during the period 1942-1943] For some time, no further work was done on the question of the independence of the axioms, but in 1950, Kamel 9 introduced a rather different axiomatization and proved its equivalence to the one given in Tarski [25]. He established the independence of some, but not all, of his axioms (see also Kamel [10]). It seems that the content of Kamel's work remained unknown to Tarski's school, although there is a reference to [10] in the bibliography of $[3]^{2}$

In a graduate topics course on relation algebras that he held at the University of California at Berkeley in 1970, Tarski talked briefly about McKinsey's result and mentioned that no further work had been done to investigate the independence of the remaining axioms. The main purpose of the present paper is to fulfill the goal implicit in Tarski's remark by demonstrating the independence of all of Tarski's axioms. The second purpose of the paper is to show that with a very minor variation in one of the axioms, namely by using the left-hand form of the distributive law for relative multiplication instead of the right-hand form, one of the remaining axioms - namely, the second involution law - does indeed become derivable from the other axioms and is therefore not independent of them. With this redundant law excluded, the resulting set of axioms is independent and is equivalent to Tarski's system. The final purpose of the paper is to show that if both the left-hand and right-hand distributive laws for relative multiplication are included in the axiom set, then two other axioms - namely, the second involution law and the distributive law for converse - become derivable from the set of axioms obtained by excluding these two laws, and therefore they are not independent. With these two redundant laws excluded, the resulting set of axioms is independent and is equivalent to Tarski's system.

The original independence models presented in this paper, with the exception of those for (R7) and (R9), were discovered "by hand", without the aid of a computer. They are different from Kamel's models. Subsequently, a systematic search, using the model searching program Mace4, developed by William McCune [19], was employed to discover the remaining two independence models, albeit in very different forms from those presented here. These models were then analyzed "by hand" in order to understand their true natures and underlying structures. Mace4 was also employed to determine the minimality of the size of some of the independence models.

There are several aspects of the paper to which we would like to draw the readers attention. First, as already mentioned, it completes the task, begun more than 70 years ago, of showing that Tarski's axiom system is independent. Second, the independence models are of some interest in their own right, and may motivate further study of classes of algebras in which, say, all but one of Tarski's axioms hold. The

\footnotetext{
${ }^{1}$ McKinsey's independence model is briefly discussed on pages $357-358$ of 18 .

${ }^{2}$ Tarski never referred to the paper in his 1970 course on relation algebras nor did he mention the paper to Givant during their long collaboration. As far as we know, with the exception of the bibliographic reference in [3]-which finds no echo in the text of [3-Kamel's work is not referred to in any other papers or books originating with members of Tarski's school and dealing with the subject of relation algebras.
} 
work of Roger Maddux can serve as a paradigm for such investigations. In a series of papers [13, 14], 15, [16], [17] (see also [18]), he has studied classes of algebras in which all of the axioms except the associative law hold, and he has established interesting and important connections between these classes of algebras and certain restricted forms of first-order logic in which there are only three variables. Third, although the independence models presented in this paper are specifically constructed for the purpose of demonstrating the independence of Tarski's axioms, modifications of these models may prove useful in establishing independence results for axiomatizations of other systems of algebraic logic, for example cylindric algebras and polyadic algebras. Fourth, future researchers may find it advantageous to use one of the alternative axiomatizations of the theory of relation algebras that are suggested in this paper. They may also find the results in the paper helpful in determining the role that each of Tarski's axioms plays in the derivation of various important relation algebraic laws. Fifth, as mentioned above, the construction of some of our independence models has been facilitated by the use of a computer, and this may resonate with computer scientists who are familiar with the use of relational methods in computer science. This applies in particular to researchers within the RAMiCS community. Finally, as the referee has pointed out, the results in this paper may serve as a salutary lesson for readers who believe that the independence of Tarski's axiom system is not very surprising. Indeed, as already mentioned, we show that if the right-hand distributive law for relative multiplication is replaced by what seems to be a harmless variant, namely the left-hand version of the law, then one of Tarski's other axioms does turn out to be redundant; and if both distributive laws are adopted as axioms, then two of Tarski's other axioms turn out to be redundant.

We are indebted to Roger Maddux for several remarks that have helped us to improve the paper, and in particular for suggesting the use of Lemma 4 (which occurs as part of Theorem 313 in [18]) in order to simplify our original proofs of Theorems 2 and 4 . We are also indebted to the referee for a very careful reading of the paper, and for several very helpful suggestions. In particular, he suggested the inclusion of the remarks in the preceding paragraph, and he also suggested the current proof of Lemma 5, which is much simpler than our original proof.

\section{Axioms and fundamental notions}

Unless explicitly stated otherwise, all algebras below have the form

$$
\mathfrak{A}=\left(A,+,-, ;,{ }^{\smile}, 1^{\prime}\right),
$$

where $A$ is a non-empty set of elements, while + and ; are binary operations on $A$, and - and ` are unary operations on $A$, and 1 ' is a distinguished constant in $A$. The set $A$ is called the universe of $\mathfrak{A}$, the Boolean operations + and - are called addition and complement respectively, and the Peircean operations ; and $`$ are called relative multiplication and converse respectively. The distinguished Peircean constant 1' is called the identity element.

Definition 1. A relation algebra is an algebra $\mathfrak{A}$ in which the following axioms are satisfied for all elements $r, s$, and $t$ in $\mathfrak{A}$.

(R1) $r+s=s+r$.

(R2) $r+(s+t)=(r+s)+t$.

(R3) $-(-r+s)+-(-r+-s)=r$. 
(R4) $r ;(s ; t)=(r ; s) ; t$.

(R5) $r ; 1^{\prime}=r$.

(R6) $r^{\smile \smile=r . ~}$

(R7) $(r ; s)^{\smile}=s^{\smile} ; r^{\smile}$.

(R8) $(r+s) ; t=r ; t+s ; t$.

(R9) $(r+s)^{\smile}=r^{\smile}+s^{\smile}$.

(R10) $r^{\smile} ;-(r ; s)+-s=-s$.

Axiom (R1) is called the commutative law for addition, (R2) is the associative law for addition, (R3) is Huntington's law, (R4) is the associative law for relative multiplication, (R5) is the right-hand identity law for relative multiplication, (R6) is the first involution law, (R7) is the second involution law, (R8) is the right-hand distributive law for relative multiplication, (R9) is the distributive law for converse, and (R10) is Tarski's law. The left-hand distributive law for relative multiplication,

$$
r ;(s+t)=r ; s+r ; t,
$$

will also play a role in our discussion.

The conventions regarding the order in which operations are to be performed are as follows: unary operations take precedence over binary operations, and among binary operations, multiplications take precedence over additions. (It is unnecessary in this paper to establish a convention regarding the order in which different unary operations are to be performed, as we shall always use parentheses to make this order clear.) For example, in fully parenthesized form, axioms (R7), (R8), and (R10) might be written as

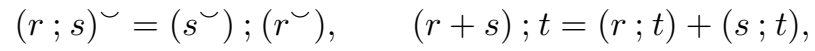

and

$$
\left(\left(r^{\smile}\right) ;(-(r ; s))\right)+(-s)=-s .
$$

Axioms (R1)-(R3) say that the Boolean part of a relation algebra $\mathfrak{A}$, namely the algebra $(A,+,-)$, is a Boolean algebra. In particular, the notions and laws from the theory of Boolean algebras apply to relation algebras. For example, the binary operation - of multiplication, and the distinguished constants 0 and 1 (called zero and the unit respectively) are defined by

$$
r \cdot s=-(-r+-s), \quad 1=1^{\prime}+-1^{\prime}, \quad 0=-1=-\left(1^{\prime}+-1^{\prime}\right) .
$$

Similarly, the partial order $\leq$ is defined by

$$
r \leq s \text { if and only if } r+s=s .
$$

The supremum, or sum, of a subset $X$ of $A$ is defined to be the least upper bound of $X$ in the sense of the partial order $\leq$, but in the case of infinite subsets, such sums may not exist. An atom is defined to be a minimal, non-zero element, and a Boolean algebra with additional operations is said to be atomic if every non-zero element is above an atom. The complement of the distinguished constant 1 ' is called the diversity element and is denoted by 0'. An atom below 0' is called a subdiversity atom. Whenever some laws of Boolean algebra are needed to justify a step in one of the proofs below, we shall simply say that the step is justified "by (the laws of) Boolean algebra".

Axioms (R4)-(R7) say that the Peircean part of a relation algebra $\mathfrak{A}$, namely the algebra $\left(A, ;,^{\smile}, 1^{\prime}\right)$, is a monoid with an involution that is an anti-isomorphism. 
Axioms (R8) and (R9) ensure that relative multiplication is distributive on the right, and converse is distributive, over addition. The binary operation ; is said to be completely distributive, or to distribute over arbitrary sums, if for all subsets $X$ and $Y$ (including infinite subsets and also the empty subset) of $\mathfrak{A}$, the existence of the sums (or suprema) $\sum X$ and $\sum Y$ implies that the sum

$$
\sum\{r ; s: r \in X \text { and } s \in Y\}
$$

exists and is equal to $\left(\sum X\right) ;\left(\sum Y\right)$. A similar definition applies to the operation of converse.

There are other versions of (R10) that are useful. For example, it is clear from the definition of the partial order $\leq$ that if $(\mathrm{R} 1)-(\mathrm{R} 3)$ are valid in a model $\mathfrak{A}$, then the validity of (R10) in $\mathfrak{A}$ is equivalent to the validity of the inequality

$$
r^{\smile} ;-(r ; s) \leq-s
$$

in $\mathfrak{A}$. We shall often make use of this equivalence in establishing the validity or failure of (R10) in a model. There is yet another form of (R10) that we shall need. If (R1)-(R3) and either $\left(\underline{\mathrm{R} 8^{\prime}}\right)$ or else (R6)-(R9) are valid in a model $\mathfrak{A}$, then the validity of (R10) in $\mathfrak{A}$ is equivalent to the validity of the implication

$$
(r ; s) \cdot t=0 \quad \text { implies } \quad\left(r^{\smile} ; t\right) \cdot s=0
$$

in $\mathfrak{A}$ (see Lemma 2 and the remark following it in Section 15). If, in addition, the model $\mathfrak{A}$ is atomic, and if the Peircean operations distribute over arbitrary sums, then the validity of (R11) (and hence also of (R10)) is equivalent to the validity of the implication (R11) for atoms. It is often convenient to use the implication (R11) in its contrapositive form:

$$
\left(r^{\smile} ; t\right) \cdot s \neq 0 \quad \text { implies } \quad(r ; s) \cdot t \neq 0 .
$$

In this form, the version of (R11) for atoms assumes the form

$$
s \leq r^{\smile} ; t \quad \text { implies } \quad t \leq r ; s
$$

for all atoms $r, s$, and $t$ in $\mathfrak{A}$.

There are two more laws that will play a role in the discussion below (see Lemma 1 in Section 15), namely the implications

$$
r \leq s \quad \text { implies } \quad r ; t \leq s ; t,
$$

and

$$
r \leq s \quad \text { implies } \quad t ; r \leq t ; s .
$$

They are respectively called the left-hand and the right-hand monotony laws for relative multiplication.

\section{ExAmples of RELATiOn ALGEBRAS}

The first task of this paper is the construction of independence models for each of Tarski's axioms (R1)-(R10). The models will often be obtained by taking wellknown relation algebras and modifying one or more of their operations in some way. In this section, we briefly describe the relation algebras that will be used to construct independence models.

The classic example motivating the entire theory of relation algebras is the algebra of all binary relations on a set $U$. The universe of this algebra is the set of 
all (binary) relations on $U$. The operations of the algebra are union, complement (with respect to the universal relation $U \times U$ ), relational composition, and converse, which are respectively defined by

$$
R \mid S=\{(\alpha, \beta):(\alpha, \gamma) \in R \text { and }(\gamma, \beta) \in S \text { for some } \gamma \in U\}
$$

and

$$
R^{-1}=\{(\alpha, \beta):(\beta, \alpha) \in R\} .
$$

The distinguished constant is the identity relation $i d_{U}$ on $U$. The algebra is called the full set relation algebra on $U$.

\begin{tabular}{c|c|c|c|c|}
$\mid$ & $\varnothing$ & $i d_{U}$ & $d i_{U}$ & $U \times U$ \\
\hline$\varnothing$ & $\varnothing$ & $\varnothing$ & $\varnothing$ & $\varnothing$ \\
\hline$i d_{U}$ & $\varnothing$ & $i d_{U}$ & $d i_{U}$ & $U \times U$ \\
\hline$d i_{U}$ & $\varnothing$ & $d i_{U}$ & $U \times U$ & $U \times U$ \\
\hline$U \times U$ & $\varnothing$ & $U \times U$ & $U \times U$ & $U \times U$ \\
\hline
\end{tabular}

TABLE 1. Relational composition table for $\mathfrak{M}_{3}$.

A more general class of examples is obtained by allowing the universe to be an arbitrary set of relations on $U$ that contains the universal relation and the identity relation, and that is closed under the operations of union, complement, relational composition, and converse. Such algebras, which are called set relation algebras, are subalgebras of the full set relation algebra on $U$. For example, fix an arbitrary set $U$ of cardinality three, and consider the set $M_{3}$ consisting of the empty relation $\varnothing$, the identity relation $i d_{U}$, the diversity relation $d i_{U}$ (the complement of the identity relation), and the universal relation $U \times U$. Certainly, $M_{3}$ is a subset of the full set relation algebra on $U$, and it contains the universal relation and the identity relation on $U$. It is clear that $M_{3}$ is closed under the Boolean operations of union and complement, and it is equally clear that $M_{3}$ is closed under the operation of converse, because every relation $R$ in $M_{3}$ is symmetric in the sense that $R^{-1}=R$. The relational composition of two relations in $M_{3}$ is again a relation in $M_{3}$, as Table 1 shows, so $M_{3}$ is closed under the operation of relational composition. Conclusion: $M_{3}$ is the universe of a set relation algebra $\mathfrak{M}_{3}$, and in fact $\mathfrak{M}_{3}$ is the minimal set relation algebra on a set of cardinality three.

Another class of examples of relation algebras may be constructed from Boolean algebras. Fix a Boolean algebra $(A,+,-)$, define relative multiplication and converse on $A$ to be the operations of Boolean multiplication and the identity function respectively, and take the identity element to be the Boolean unit, so that

$$
r ; s=r \cdot s, \quad r^{\smile}=r, \quad 1^{\prime}=1
$$

for all $r$ and $s$. The resulting algebra $\mathfrak{A}$ is easily seen to be a relation algebra, and it is called a Boolean relation algebra. A concrete instance of this construction is provided by the two-element Boolean algebra, whose universe consists of the elements 0 and 1.

A third class of examples of relation algebras may be constructed from groups. Fix a group

$$
(G, \circ,-1, \iota)
$$


with a binary composition operation ${ }^{\circ}$, a unary inverse operation ${ }^{-1}$, and an identity element $\iota$. Take $A$ to be the set of all subsets of $G$. Obviously, $A$ is closed under arbitrary unions and under complements (formed with respect to $G$ ). Define operations ; and $\smile$ of complex multiplication and complex inverse by

$$
X ; Y=\{f \circ g: f \in X \text { and } g \in Y\} \quad \text { and } \quad X^{\smile}=\left\{f^{-1}: f \in X\right\}
$$

for all subsets $X$ and $Y$ of $G$, and take the distinguished element 1 ' to be the singleton of the group identity element, $\{\iota\}$. The resulting algebra $\mathfrak{A}$ (in which addition and complement are defined to be the set-theoretic operations of union and complement) is a relation algebra, as was shown by McKinsey some time in the 1940 s (see [7]). It is called the complex algebra of the group $G$. For a concrete instance of this construction, take $G$ to be the additive group of integers modulo 3 . The operations of relative multiplication and converse in the complex algebra of this group are set forth in Tables 2 and 3 respectively.

\begin{tabular}{|c|c|c|c|c|c|c|c|c|}
\hline$;$ & $\varnothing$ & $\{0\}$ & $\{1\}$ & $\{2\}$ & $\{0,1\}$ & $\{0,2\}$ & $\{1,2\}$ & $\{0,1,2\}$ \\
\hline$\varnothing$ & $\varnothing$ & $\varnothing$ & $\varnothing$ & $\varnothing$ & $\varnothing$ & $\varnothing$ & $\varnothing$ & $\varnothing$ \\
\hline$\{0\}$ & $\varnothing$ & $\{0\}$ & $\{1\}$ & $\{2\}$ & $\{0,1\}$ & $\{0,2\}$ & $\{1,2\}$ & $\{0,1,2\}$ \\
\hline$\{1\}$ & $\varnothing$ & $\{1\}$ & $\{2\}$ & $\{0\}$ & $\{1,2\}$ & $\{0,1\}$ & $\{0,2\}$ & $\{0,1,2\}$ \\
\hline$\{2\}$ & $\varnothing$ & $\{2\}$ & $\{0\}$ & $\{1\}$ & $\{0,2\}$ & $\{1,2\}$ & $\{0,1\}$ & $\{0,1,2\}$ \\
\hline$\{0,1\}$ & $\varnothing$ & $\{0,1\}$ & $\{1,2\}$ & $\{0,2\}$ & $\{0,1,2\}$ & $\{0,1,2\}$ & $\{0,1,2\}$ & $\{0,1,2\}$ \\
\hline$\{0,2\}$ & $\varnothing$ & $\{0,2\}$ & $\{0,1\}$ & $\{1,2\}$ & $\{0,1,2\}$ & $\{0,1,2\}$ & $\{0,1,2\}$ & $\{0,1,2\}$ \\
\hline$\{1,2\}$ & $\varnothing$ & $\{1,2\}$ & $\{0,2\}$ & $\{0,1\}$ & $\{0,1,2\}$ & $\{0,1,2\}$ & $\{0,1,2\}$ & $\{0,1,2\}$ \\
\hline$\{0,1,2\}$ & $\varnothing$ & $\{0,1,2\}$ & $\{0,1,2\}$ & $\{0,1,2\}$ & $\{0,1,2\}$ & $\{0,1,2\}$ & $\{0,1,2\}$ & $\{0,1,2\}$ \\
\hline
\end{tabular}

TABLE 2. Relative multiplication table for the complex algebra of the group of integers modulo 3 .

\begin{tabular}{|c|c|}
\hline$r$ & $r^{\smile}$ \\
\hline$\varnothing$ & $\varnothing$ \\
\hline$\{0\}$ & $\{0\}$ \\
\hline$\{1\}$ & $\{2\}$ \\
\hline$\{2\}$ & $\{1\}$ \\
\hline$\{0,1\}$ & $\{0,2\}$ \\
\hline$\{0,2\}$ & $\{0,1\}$ \\
\hline$\{1,2\}$ & $\{1,2\}$ \\
\hline$\{0,1,2\}$ & $\{0,1,2\}$ \\
\hline
\end{tabular}

TABLE 3. Converse table for the complex algebra of the group of integers modulo 3 .

For the fourth and final example of a relation algebra, consider the eight-element Boolean algebra $(D,+,-)$ with three atoms, say 1 ', $a$, and $b$. Define an operation of relative multiplication on these atoms as in Table 4, and extend this operation to all of $D$ by requiring it to be distributive over arbitrary sums (see Table 5, and keep in mind that $\left.0^{\prime}=-1^{\prime}=a+b\right)$. Take converse to be the identity function on 
$D$. Lyndon [12] was the first to observe the resulting algebra $\mathfrak{D}$ is a relation algebra. (This algebra is discussed on page 429 of Maddux [18], where it is denoted by $7_{7}$; see, in particular, Table 34.) In fact, $\mathfrak{D}$ can be represented as a set relation algebra, and also as a subalgebra of the complex algebra of the group $G \times G$, where $G$ is the additive group of integers modulo 3 , but these observations will not play a role in the discussion below.

\begin{tabular}{c|c|c|c|}
$;$ & $1^{\prime}$ & $a$ & $b$ \\
\hline $1^{\prime}$ & $1^{\prime}$ & $a$ & $b$ \\
\hline$a$ & $a$ & 1 & $0^{\prime}$ \\
\hline$b$ & $b$ & $0^{\prime}$ & 1 \\
\hline
\end{tabular}

TABLE 4. Relative multiplication table for the atoms in $\mathfrak{D}$

\begin{tabular}{|c|c|c|c|c|c|c|c|c|}
\hline$;$ & 0 & $1^{\prime}$ & $a$ & $b$ & $1^{\prime}+a$ & $1^{\prime}+b$ & 0 & 1 \\
\hline 0 & 0 & 0 & 0 & 0 & 0 & 0 & 0 & 0 \\
\hline $1^{\prime}$ & 0 & $1^{\prime}$ & $a$ & $b$ & $1^{\prime}+a$ & $1^{\prime}+b$ & 0 & 1 \\
\hline$a$ & 0 & $a$ & 1 & $0{ }^{\prime}$ & 1 & $0^{\prime}$ & 1 & 1 \\
\hline$b$ & 0 & $b$ & $0^{\prime}$ & 1 & 0 & 1 & 1 & 1 \\
\hline $1^{\prime}+a$ & 0 & $1^{\prime}+a$ & 1 & 0 & 1 & 1 & 1 & 1 \\
\hline $1^{\prime}+b$ & 0 & $1^{\prime}+b$ & 0 & 1 & 1 & 1 & 1 & 1 \\
\hline $0^{\prime}$ & 0 & $0^{\prime}$ & 1 & 1 & 1 & 1 & 1 & 1 \\
\hline 1 & 0 & 1 & 1 & 1 & 1 & 1 & 1 & 1 \\
\hline
\end{tabular}

TABLE 5. Relative multiplication table for $\mathfrak{D}$.

\section{INDEPENDENCE}

A mathematical statement $\varphi$ is said to be independent of a set of mathematical statements $\Phi$ (with respect to a given logical framework) if $\varphi$ cannot be derived from $\Phi$ (within the given logical framework). A set of axioms $\Phi$ is said to be independent if each $\varphi$ in $\Phi$ is independent of the set of axioms obtained from $\Phi$ by removing $\varphi$. In other words, $\Phi$ is independent if none of the axioms in $\Phi$ can be derived from the remaining axioms in $\Phi$. The standard way of establishing the independence of a set of axioms is to construct for each axiom $\varphi$, a model in which $\varphi$ fails and the remaining axioms are valid. Such a model is called an independence model for $\varphi$. The first task of the present paper is to prove the following theorem.

Theorem 1. The set of Tarski's axioms (R1)-(R10) is independent.

The proof proceeds by constructing for each $n=1, \ldots, 10$ an independence $\operatorname{model} \mathfrak{A}_{n}$ for $(\mathrm{R} n)$.

\section{INDEPENDENCE OF (R1)}

Let $\left(G,{ }^{\circ},-1, \iota\right)$ be any Boolean group of order at least two, with identity element $\iota$, that is to say, any group with at least two elements in which each 
element $r$ is its own inverse, so that $r^{-1}=r$. Let addition be the binary operation of left-hand projection on $G$, which is defined by

$$
r+s=r
$$

for all $r$ and $s$ in $G$, take complement to be the identity operation on $G$, and take relative multiplication, converse, and the identity element to coincide with the corresponding group operations and identity element, so that

$$
r ; s=r \circ s, \quad r^{\smile}=r^{-1}=r, \quad \text { and } \quad 1^{\prime}=\iota
$$

for all $r$ and $s$ in $G$. In the resulting algebra $\mathfrak{A}_{1}$ (of the same similarity type as relation algebras), it is clear that (R1) fails. Indeed, for distinct elements $r$ and $s$, we have

$$
r+s=r \neq s=s+r .
$$

The sum of any finite sequence of elements in $\mathfrak{A}_{1}$ is always the left-most element in the sequence, by the definition of addition, so the associative law (R2) holds automatically; in more detail,

$$
r+(s+t)=r=r+t=(r+s)+t .
$$

Also Huntington's law (R3) holds:

$$
-(-r+s)+-(-r+-s)=(r+s)+(r+s)=r,
$$

by the definitions of complement and addition. Axioms (R4)-(R7) hold trivially in $\mathfrak{A}_{1}$, because the Peircean part of $\mathfrak{A}_{1}$ is, by definition, a Boolean group, and Boolean groups are always commutative. The distributive law (R8) holds in $\mathfrak{A}_{1}$, because

$$
(r+s) ; t=r ; t=r ; t+s ; t,
$$

by the definition of addition. The distributive law (R9) holds trivially in $\mathfrak{A}_{1}$, since converse is the identity operation. Also, Tarski's law (R10) holds in $\mathfrak{A}_{1}$, because

$$
\begin{aligned}
r^{\smile} ;-(r ; s)+-s=r^{\smile} ;(r ; s)+s & =r^{\smile} ;(r ; s) \\
& =r^{-1} \circ(r \circ s)=\left(r^{-1} \circ r\right) \circ s=\iota \circ s=s=-s .
\end{aligned}
$$

These equalities use the fact that complement is the identity operation on $G$ and addition is the left-hand projection, while converse, relative multiplication, and the identity element in $\mathfrak{A}_{1}$ coincide with the corresponding group operations and identity element, by definition.

A minimal independence model for (R1) is obtained by starting with the twoelement additive Boolean group of integers modulo 2.

\section{INDEPENDENCE OF (R2)}

The independence model $\mathfrak{A}_{2}$ is defined as follows. The universe consists of three elements, 0,1 ', and 1, and the operations of addition, relative multiplication, and complement are determined as in Table [6] Converse is defined to be the identity function on the universe, and the identity element is defined to be 1'. The associative law (R2) is easily shown to fail in $\mathfrak{A}_{2}$ : just take $r, s$, and $t$ to be $1^{\prime}, 1^{\prime}$, and 1 respectively to arrive at

$$
r+(s+t)=1^{\prime}+\left(1^{\prime}+1\right)=1^{\prime}+0=1^{\prime}
$$




\begin{tabular}{c|c|c|c|}
+ & 0 & $1^{\prime}$ & 1 \\
\hline 0 & 0 & $1^{\prime}$ & 1 \\
\hline $1^{\prime}$ & $1^{\prime}$ & $1^{\prime}$ & 0 \\
\hline 1 & 1 & 0 & 1 \\
\hline
\end{tabular}

\begin{tabular}{c|c|c|c|}
$;$ & 0 & $1^{\prime}$ & 1 \\
\hline 0 & 0 & 0 & 0 \\
\hline $1^{\prime}$ & 0 & $1^{\prime}$ & 1 \\
\hline 1 & 0 & 1 & $1^{\prime}$ \\
\hline
\end{tabular}

\begin{tabular}{c|c|}
$r$ & $-r$ \\
\hline 0 & 0 \\
\hline $1 '$ & 1 \\
\hline 1 & $1 '$ \\
\hline
\end{tabular}

TABLE 6. Addition, relative multiplication, and complement tables for the algebra $\mathfrak{A}_{2}$.

and

$$
(r+s)+t=\left(1^{\prime}+1^{\prime}\right)+1=1^{\prime}+1=0 .
$$

Turn now to the task of verifying the remaining axioms in $\mathfrak{A}_{2}$. Axioms (R6) and (R9) are valid because converse is defined to be the identity function; and (R5) is clearly valid, as a glance at the column for 1' in the relative multiplication table for $\mathfrak{A}_{2}$ shows (see Table 6). Axiom (R1) is valid because the operation table for addition is symmetric across the diagonal, and therefore addition is commutative. Similarly, (R7) is valid because the operation table for relative multiplication is symmetric across the diagonal - so that relative multiplication is commutative - and converse is the identity function. It remains to check (R3), (R4), (R8), and (R10).

Begin with the verification of (R3). If $r$ is 0 , then

$$
\begin{aligned}
-(-r+s)+-(-r & +-s)=-(-0+s)+-(-0+-s) \\
& =-(0+s)+-(0+-s)=-s+-(-s)=-s+s=0=r,
\end{aligned}
$$

by the definitions of complement and addition, and the fact that the sum of any element and its complement is always 0 in $\mathfrak{A}_{2}$ (see Table 6). Similarly, if $s$ is 0 , then

$$
\begin{aligned}
&-(-r+s)+-(-r+-s)=-(-r+0)+-(-r+-0) \\
&=-(-r)+-(-r)=r .
\end{aligned}
$$

Assume now that $r$ and $s$ are both non-zero. If $r=s$, then

$$
-(-r+s)+-(-r+-s)=-(-r+r)+-(-r+-r)=-0+-(-r)=r .
$$

The second equality uses two properties of addition in $\mathfrak{A}_{2}$ : it is an idempotent operation in the sense that $t+t=t$ for all $t$; and the sum of an element and its complement is always 0 . If $r$ and $s$ are distinct, then $r=-s$ (because $\mathfrak{A}_{2}$ has just two non-zero elements, and they are the complements of one another), and consequently (R3) is valid for $r$ and $-s$, by the case just considered. It follows that

$$
\begin{aligned}
-(-r+s)+-(-r+-s)=-(-r+ & -s)+-(-r+s) \\
& =-(-r+-s)+-(-r+-(-s))=r,
\end{aligned}
$$

by the commutativity of addition, the fact that $-(-s)=s$, and the validity of (R3) for $r$ and $-s$.

As regards the associative law (R4), if at least one of the elements $r, s$, and $t$ is 0 , then both sides of (R4) reduce to 0 , by the definition of relative multiplication. Similarly, if at least one of the three elements is 1', then both sides of (R4) reduce to the relative product of the other two elements. For example, if $s$ is 1', then

$$
r ;(s ; t)=r ;\left(1^{\prime} ; t\right)=r ; t \quad \text { and } \quad(r ; s) ; t=\left(r ; 1^{\prime}\right) ; t=r ; t .
$$


The only other possibility is that all three elements are 1 , and in this case both sides of (R4) reduce to 1 .

Turn next to the verification of (R8). If $t$ is 0 , then both sides of (R8) reduce to 0 , and if $t$ is 1 ', then both sides reduce to $r+s$, by the definition of relative multiplication. Similarly, if $r$ is 0 , then both sides of (R8) reduce to $s ; t$, and if $s$ is 0 or if $r=s$, then both sides reduce to $r ; t$. There remain the cases when $t$ is 1 , and $r$ and $s$ are distinct values in the set $\left\{1^{\prime}, 1\right\}$. In this case, $r+s$ is 0 , by the definition of addition, so the left side of $(\mathrm{R} 8)$ reduces to 0 ; and the right side of $(\mathrm{R} 8)$ reduces to $1^{\prime} ; 1+1 ; 1$, which is also 0 .

To verify (R10), observe first that $1 ; s=-s$ for any value of $s$ in $\mathfrak{A}_{2}$, by the definitions of relative multiplication and complement (see Table 6). If $r$ is 0 , then

$$
\begin{aligned}
r^{\smile} ;-(r ; s)+-s=0^{\smile} ;-(0 ; s)+-s=0 ;-0 & -s \\
& =0 ; 0+-s=0+-s=-s,
\end{aligned}
$$

if $r$ is 1 , then

$$
r^{\smile} ;-(r ; s)+-s=1^{\smile \smile} ;-\left(1^{\prime} ; s\right)+-s=1^{\prime} ;-s+-s=-s,
$$

and if $r$ is 1 , then

$$
r^{\smile} ;-(r ; s)+-s=1^{\smile} ;-(1 ; s)+-s=1 ;-(-s)+-s=1 ; s+-s=-s .
$$

Thus, in all three cases, the left side of (R10) reduces to $-s$, as desired.

A computational check using the model searching program Mace4 has shown that $\mathfrak{A}_{2}$ is the unique independence model for (R2) of cardinality 3 , and there is no independence model for (R2) of smaller cardinality.

\section{IndePENDENCE of (R3)}

Consider any Boolean relation algebra with at least two elements. Modify the definition of complement in this algebra by requiring it to be the identity function, that is to say, by requiring $-r=r$ for all $r$. In the resulting algebra $\mathfrak{A}_{3}$, axiom (R3) fails, because

$$
-(-r+s)+-(-r+-s)=r+s
$$

by the definition of complement, and $r+s$ is different from $r$ whenever the element $s$ is not below $r$ (as is the case if, say, $s$ is 1 and $r$ is less than 1). Axioms (R1), (R2), and (R4)-(R9) all hold in $\mathfrak{A}_{3}$, because they do not involve the operation of complement. Also, (R10) holds in $\mathfrak{A}_{3}$, because

$$
r^{\smile} ;-(r ; s)+-s=r \cdot(r \cdot s)+s=s=-s,
$$

by the definitions of the operations of converse, relative multiplication, and complement.

A minimal independence model for (R3) is obtained by starting with a Boolean relation algebra of cardinality 2 .

\section{INDEPENDENCE OF (R4)}

To construct an independence model for (R4), start with a three-element partial algebra $(G, \circ,-1, \iota)$ of the same similarity type as a group. The universe $G$ of this partial algebra is the set $\{0,1,2\}$, the binary partial operation $\circ$ is determined by Table 7 , the unary operation ${ }^{-1}$ is the identity function on $G$, and the distinguished constant $\iota$ is 0 . The values of $1 \circ 2$ and $2 \circ 1$ in the operation table for $\circ$ are left 


\begin{tabular}{|l|l|l|l|}
\hline$\circ$ & 0 & 1 & 2 \\
\hline 0 & 0 & 1 & 2 \\
\hline 1 & 1 & 0 & \\
\hline 2 & 2 & & 0 \\
\hline
\end{tabular}

TABLE 7. Table for the operation $\circ$.

undefined. Form the complex algebra $\mathfrak{A}_{4}$ of this partial algebra in exactly the same way as the complex algebras of groups are formed. The operation of relative multiplication in $\mathfrak{A}_{4}$ is given by Table 8 . Converse is the identity function on the universe of $\mathfrak{A}_{4}$, and $\{0\}$ is the identity element with respect to the operation of relative multiplication.

\begin{tabular}{|c|c|c|c|c|c|c|c|c|}
\hline$;$ & $\varnothing$ & $\{0\}$ & $\{1\}$ & $\{2\}$ & $\{0,1\}$ & $\{0,2\}$ & $\{1,2\}$ & $\{0,1,2\}$ \\
\hline$\varnothing$ & $\varnothing$ & $\varnothing$ & $\varnothing$ & $\varnothing$ & $\varnothing$ & $\varnothing$ & $\varnothing$ & $\varnothing$ \\
\hline$\{0\}$ & $\varnothing$ & $\{0\}$ & $\{1\}$ & $\{2\}$ & $\{0,1\}$ & $\{0,2\}$ & $\{1,2\}$ & $\{0,1,2\}$ \\
\hline$\{1\}$ & $\varnothing$ & $\{1\}$ & $\{0\}$ & $\varnothing$ & $\{0,1\}$ & $\{1\}$ & $\{0\}$ & $\{0,1\}$ \\
\hline$\{2\}$ & $\varnothing$ & $\{2\}$ & $\varnothing$ & $\{0\}$ & $\{2\}$ & $\{0,2\}$ & $\{0\}$ & $\{0,2\}$ \\
\hline$\{0,1\}$ & $\varnothing$ & $\{0,1\}$ & $\{0,1\}$ & $\{2\}$ & $\{0,1\}$ & $\{0,1,2\}$ & $\{0,1,2\}$ & $\{0,1,2\}$ \\
\hline$\{0,2\}$ & $\varnothing$ & $\{0,2\}$ & $\{1\}$ & $\{0,2\}$ & $\{0,1,2\}$ & $\{0,2\}$ & $\{0,1,2\}$ & $\{0,1,2\}$ \\
\hline$\{1,2\}$ & $\varnothing$ & $\{1,2\}$ & $\{0\}$ & $\{0\}$ & $\{0,1,2\}$ & $\{0,1,2\}$ & $\{0\}$ & $\{0,1,2\}$ \\
\hline$\{0,1,2\}$ & $\varnothing$ & $\{0,1,2\}$ & $\{0,1\}$ & $\{0,2\}$ & $\{0,1,2\}$ & $\{0,1,2\}$ & $\{0,1,2\}$ & $\{0,1,2\}$ \\
\hline
\end{tabular}

TABLE 8. Operation table for relative multiplication in $\mathfrak{A}_{4}$.

To see that (R4) fails in $\mathfrak{A}_{4}$, take $r$ to be the atom $\{1\}$, and take $s$ and $t$ to be the atom $\{2\}$, to obtain

$$
r ;(s ; t)=\{1\} ;(\{2\} ;\{2\})=\{1\} ;\{0\}=\{1\},
$$

and

$$
(r ; s) ; t=(\{1\} ;\{2\}) ;\{2\}=\varnothing ;\{2\}=\varnothing .
$$

The Boolean part of $\mathfrak{A}_{4}$ is, by definition, a Boolean algebra of sets, so (R1)-(R3) are certainly valid in $\mathfrak{A}_{4}$. The operation of relative multiplication is commutative and distributive over addition in $\mathfrak{A}$, because $\circ$ is a commutative partial operation (see Table 7), and because the very definition of the complex operation ; in terms of - (see Section 3) implies that it distributes over arbitrary sums. From these observations, together with the fact that converse is the identity function on the universe of $\mathfrak{A}_{4}$, and $\{0\}$ is an identity element with respect to operation of relative multiplication, it follows that (R5)-(R9) all hold trivially in $\mathfrak{A}_{4}$.

It remains to show that (R10) is valid in $\mathfrak{A}_{4}$. We do this by verifying condition (R11) for atoms in its contrapositive form (see the remarks at the end of Section 21). In the present situation, this amounts to checking that

$$
s \leq r ; t \quad \text { implies } \quad t \leq r ; s
$$

for all atoms $r, t$, and $s$. If $r$ is the identity element $\{0\}$, then (1) reduces to the triviality that $s=t$ implies $t=s$. If $t$ is the identity element, then the hypothesis 
of (11) reduces to $s=r$; in this case $r ; s=r ; r$, which is always the identity element when $r$ is an atom (see Table 8), so the conclusion of (11) holds. We may therefore assume that $r$ and $t$ are atoms distinct from the identity element. If $r=t$, then the hypothesis of (11) is only satisfied if $s$ is the identity element (see Table 8), and in this case the conclusion of (1) holds trivially. The only remaining case is when $r$ and $t$ are, in some order, the two subdiversity atoms $\{1\}$ and $\{2\}$. In this case the relative product $r ; t$ is the empty set (see Table 8), so the hypothesis of (1) is never satisfied, and therefore the implication in (1) is always true.

The algebra $\mathfrak{A}_{4}$ was discovered by J. C. C. McKinsey some time in the early 1940s. A computational check using Mace4 has shown that there is no independence model for (R4) of smaller cardinality. Roger Maddux has studied variants of relation algebras in which only weakened versions of the associative law hold. In particular, he has constructed numerous examples of algebras in which (R4) fails and the rest of Tarski's axioms hold; see in particular Theorems 2.5(3), 2.5(4), 3.7, and 3.10 in [14] and see also [18.

\section{INDEPENDENCE OF (R5)}

Consider any Boolean algebra $(A,+,-)$ with at least two elements. Define relative multiplication to be the binary operation on $A$ whose value on any two arguments is always 0 , so that

$$
r ; s=0
$$

for all $r$ and $s$. Take converse to be the identity function on $A$, and take $1^{\prime}$ to be any element in $A$. In the resulting algebra $\mathfrak{A}_{5}$,

$$
r ; 1^{\prime}=0 \neq r
$$

whenever $r$ is a non-zero element, so (R5) fails. The Boolean axioms (R1)-(R3) obviously hold in $\mathfrak{A}_{5}$, and the associative law (R4) for relative multiplication holds because both sides of (R4) reduce to 0 . The same is true of (R7),

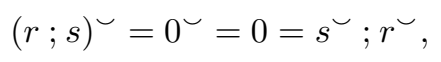

and (R8),

$$
(r+s) ; t=0=0+0=r ; t+s ; t .
$$

Axioms (R6) and (R9) hold trivially because converse is the identity function, and Tarski's law (R10) holds because

$$
r^{\smile} ;-(r ; s)+-s=0+-s=-s .
$$

There is another, rather trivial independence model for (R5) that should be mentioned. Consider any relation algebra $\mathfrak{B}$ with at least two elements. The identity element in $\mathfrak{B}$ is uniquely determined in the sense that there is exactly one element in $\mathfrak{B}$ for which (R5) holds (see Theorem 1.2 in Chin-Tarski [1). Take $\mathfrak{B}_{5}$ to be the algebra obtained from $\mathfrak{B}$ by choosing 1 ' to be any element different from the identity element in $\mathfrak{B}$ (for example, choose $1^{\prime}$ to be the zero element in $\mathfrak{B}$ ). Axiom (R5) fails in $\mathfrak{B}_{5}$, because $1^{\prime}$ is not the identity element in $\mathfrak{B}$. But (R1)-(R4) and (R6)-(R10) all hold in $\mathfrak{B}_{5}$, because they hold in $\mathfrak{B}$ and they do not explicitly mention 1'.

The independence model $\mathfrak{B}_{5}$ has one important defect. There is a formulation of (R5) that is not equational, but rather existential in form and does not utilize a distinguished constant; instead, it asserts the existence of a right-hand identity element for relative multiplication (see, for example, Chin-Tarski [1]). This existential 
form of (R5) is true in $\mathfrak{B}_{5}$, so $\mathfrak{B}_{5}$ cannot be used to demonstrate the independence of the existential form of (R5) from the remaining axioms. On the other hand, $\mathfrak{A}_{5}$ can still be used for this purpose.

A minimal independence model for (R5) may be obtained by using the twoelement Boolean algebra to construct $\mathfrak{A}_{5}$.

\section{INDEPENDENCE OF (R6)}

Let $\mathfrak{A}$ be any relation algebra with at least two elements, and modify the definition of converse by requiring

$$
r^{\smile}=0
$$

for all $r$. Obviously, (R6) fails in the resulting algebra $\mathfrak{A}_{6}$, since for any non-zero element $r$ we have

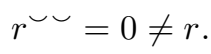

It is equally clear (R1)-(R5) and (R8) are valid in $\mathfrak{A}_{6}$, because these axioms are valid in $\mathfrak{A}$ and do not contain any occurrence of converse. Also, (R7) is valid in $\mathfrak{A}_{6}$, because

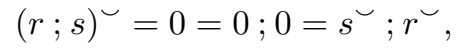

by the definition of converse, and the fact that relative multiplication by 0 always yields 0 in the relation algebra $\mathfrak{A}$, and hence also in $\mathfrak{A}_{6}$. A similar argument shows that (R9) is valid in $\mathfrak{A}_{6}$, because both sides of the axiom reduce to 0 . Finally, to verify (R10), or equivalently, (R10'), in $\mathfrak{A}_{6}$, observe that

$$
r^{\smile} ;-(r ; s)=0 ;-(r ; s)=0 \leq-s,
$$

by the definition of converse, and the fact that relative multiplication by 0 always yields 0 in $\mathfrak{A}$, and hence also in $\mathfrak{A}_{6}$.

A minimal independence model for (R6) may be obtained by taking $\mathfrak{A}$ to be the two-element Boolean relation algebra.

\section{IndePENDENCE of (R7)}

Let $(A,+,-)$ be any Boolean algebra with at least four elements, and take 1 ' to be any element in $A$ that is different from 0 and 1 . For instance, 1' might be an atom. Define a binary operation ; on $A$ by modifying slightly the definition of relative multiplication in the independence model $\mathfrak{A}_{5}$ for $(\mathrm{R} 5)$ :

$$
r ; s=\left\{\begin{array}{ll}
r & \text { if } \quad s=1, \\
0 & \text { if } \quad s \neq 1
\end{array},\right.
$$

for all $r$ and $s$. Take $\smile$ to be the identity function on $A$.

Axiom (R7) fails in the resulting algebra $\mathfrak{A}_{7}$ because relative multiplication is not commutative. In more detail, if $r$ is an element different from 0 and 1', and if $s$ is 1 , then

$$
(r ; s)^{\smile}=r ; 1^{\prime}=r \neq 0=1^{\prime} ; r=s^{\smile} ; r^{\smile} .
$$

To see that (R4) is valid in $\mathfrak{A}_{7}$, observe that if $t=1$, then both sides of (R4) reduce to $r ; s$, and if $t \neq 1$ ', then both sides of ( $\mathrm{R} 4)$ reduce to 0 , by the definition of relative multiplication. The argument that (R8) holds is similar: if $t=1$, then both sides of (R8) reduce to $r+s$, and if $t \neq 1$, then both sides reduce to 0 . Each of (R5), (R6), and (R9) holds trivially in $\mathfrak{A}_{7}$, by the definitions of relative multiplication and converse. The verification of $(\mathrm{R} 10)$, in the form of $\left(\underline{\mathrm{R} 10^{\prime}}\right)$, in $\mathfrak{A}_{7}$ 
breaks into cases, and the argument in each case is based on the definition of converse and relative multiplication, but uses also laws of Boolean algebra in the final step. If $s \neq 1$, then

$$
r^{\smile} ;-(r ; s)=r ;-0=r ; 1=0 \leq-s .
$$

If $s=1^{\prime}$, then either $r \neq 0^{\prime}$, in which case $-r \neq 1^{\prime}$, and therefore

$$
r^{\smile} ;-(r ; s)=r ;-\left(r ; 1^{\prime}\right)=r ;-r=0 \leq-s,
$$

or else $r=0^{\prime}$, in which case $-r=1$, and therefore

$$
r^{\smile} ;-(r ; s)=0^{\prime} ;-\left(0^{\prime} ; 1^{\prime}\right)=0^{\prime} ;-0^{\prime}=0^{\prime} ; 1^{\prime}=0^{\prime}=-1^{\prime}=-s .
$$

A computational check using Mace4 has shown that there is no independence model for (R7) of smaller cardinality.

\section{INDEPENDENCE OF (R8)}

Let $\mathfrak{A}$ be any symmetric, integral relation algebra, that is to say, any relation algebra with at least two elements in which

$$
r ; s=0 \quad \text { implies } \quad r=0 \quad \text { or } \quad s=0,
$$

and in which converse is the identity function on $A$. It is well known and easy to see that the operation of relative multiplication in such a relation algebra is commutative, and for any non-zero element $r$,

$$
r ; 1=1 ; r=1
$$

(see [8]). The independence model $\mathfrak{A}_{8}$ is obtained from $\mathfrak{A}$ by changing the definition of relative multiplication in one instance, namely when both arguments are 0 , and in this case putting

$$
0 ; 0=1 .
$$

It is not difficult to check that (R8) fails in $\mathfrak{A}_{8}$ : just take $r$ and $t$ to be 0 , and $s$ to be 1', and observe that

$$
(r+s) ; t=\left(0+1^{\prime}\right) ; 0=1^{\prime} ; 0=0,
$$

but

$$
r ; t+s ; t=0 ; 0+1^{\prime} ; 0=1+0=1 .
$$

The axioms of relation algebra are valid in $\mathfrak{A}$, by assumption. Every instance of an axiom that does not involve a computation of $0 ; 0$ yields the same result in $\mathfrak{A}_{8}$ as it does in $\mathfrak{A}$, so it must hold in $\mathfrak{A}_{8}$. In particular, (R1)-(R3), (R5), (R6), and (R9) all hold in $\mathfrak{A}_{8}$. Similarly, all instances of (R7) in which $r$ and $s$ are not both 0 hold in $\mathfrak{A}$ and therefore in $\mathfrak{A}_{8}$; and when both $r$ and $s$ are 0 , each side of (R7) reduces to 1 . Thus, (R7) is valid in $\mathfrak{A}_{8}$. It remains to check the validity of (R4) and (R10).

Every instance of (R4) in which at most one of $r, s$, and $t$ is 0 must hold in $\mathfrak{A}_{8}$, since no such instance can involve a computation of $0 ; 0$. (Here, the assumption that $\mathfrak{A}$ is integral plays a role.) If all three of these elements are 0 , then the computations

$$
0 ;(0 ; 0)=0 ; 1=0 \quad \text { and } \quad(0 ; 0) ; 0=1 ; 0=0
$$


show that (R4) holds in $\mathfrak{A}_{8}$ in this case as well. There remain the three cases when exactly two of the elements are 0 . If $r$ and $s$ are both 0 , and $t$ is different from 0 , then

$$
r ;(s ; t)=0 ; 0=1 \quad \text { and } \quad(r ; s) ; t=1 ; t=1
$$

(the assumption that $\mathfrak{A}$ is integral justifies the last step). A similar argument applies when $s$ and $t$ are 0 , and $r$ is different from 0 . If $r$ and $t$ are 0 , and $s$ is different from 0 , then

$$
r ;(s ; t)=0 ; 0=1 \quad \text { and } \quad(r ; s) ; t=0 ; 0=1 .
$$

Conclusion: (R4) is valid in $\mathfrak{A}_{8}$.

There is only one instance of (R10) that involves a computation of $0 ; 0$, namely when $r$ and $s$ are both 0 . In this case, and in every other case in which $s$ is 0 , we have $-s=1$, so the two sides of (R10) evaluate to 1 . Since the remaining instances of (R10) do not lead to a computation involving $0 ; 0$, they automatically hold in $\mathfrak{A}_{8}$. For example, suppose $r$ and $s$ are not both 0 , but $-(r ; s)$ is 0 . In this case, $r ; s$ must be 1 , so $r$-and therefore also $r^{\smile}$-must be different from 0 . Consequently, this instance of (R10) does not involve a computation of $0 ; 0$, and therefore it holds in $\mathfrak{A}_{8}$.

Take $\mathfrak{A}$ to be the two-element Boolean relation algebra to arrive at a minimal independence model for (R8).

\section{INDEPENDENCE OF (R9)}

Let $\mathfrak{C}$ be the complex algebra of the additive group of integers modulo 3 (see Section 2, and in particular Table 2). In order to avoid notational confusion, write 。 and ${ }^{-1}$ for the operations of relative multiplication (composition of complexes) and converse (inversion of complexes) in $\mathfrak{C}$, and write ; and $\smile$ for the corresponding operations in $\mathfrak{A}_{9}$. The independence model $\mathfrak{A}_{9}$ for $(\mathrm{R} 9)$ is obtained from $\mathfrak{C}$ by changing the definitions of converse and relative multiplication slightly, while leaving the remaining operations intact. In fact, the table for converse in $\mathfrak{A}_{9}$ is obtained from the table for converse in $\mathfrak{C}$ by changing the value of converse on the two singletons $\{1\}$ and $\{2\}$, while leaving its value on the remaining elements unchanged. In $\mathfrak{C}$, converse interchanges these two singletons, whereas in $\mathfrak{A}_{9}$ converse is defined to map each of these singletons to itself. Put somewhat differently, converse in $\mathfrak{A}_{9}$ maps every element to itself, with the exception of the two doubletons $\{0,1\}$ and $\{0,2\}$, which it interchanges. As a result, (R9) must fail in $\mathfrak{A}_{9}$. Indeed, if $r$ and $s$ are taken to be $\{0\}$ and $\{2\}$ respectively, then

$$
(r+s)^{\smile}=(\{0\}+\{2\})^{\smile}=\{0,2\}^{\smile}=\{0,1\} \neq\{0\}+\{2\}=r^{\smile}+s^{\smile} .
$$

Unfortunately, this change in the definition of converse causes other axioms to fail, for example (R7). In order to avoid this undesired side effect, the operation of relative multiplication must also be altered in the passage from $\mathfrak{C}$ to $\mathfrak{A}_{9}$. Specifically, it is altered in the six cases that involve relative multiplication of one of the two singletons $\{1\}$ and $\{2\}$ on the left with one of the three doubletons on the right. If $r$ is any singleton, and $s$ any doubleton, then the relative product $r ; s$ in $\mathfrak{A}_{9}$ is defined to coincide with the relative product of $r^{-1}$ and $s$ in $\mathfrak{C}$, in symbols

$$
r ; s=r^{-1} \circ s .
$$


This has the effect of interchanging the relevant parts of the rows for $\{1\}$ and $\{2\}$ in the operation table for relative multiplication in $\mathfrak{C}$, but leaving the row for $\{0\}$ unchanged (see Tables 2 and 9). In particular, relative multiplication by $\{0\}$ in $\mathfrak{A}_{9}$

\begin{tabular}{|c|c|c|c|}
\hline$\circ$ & $\{0,1\}$ & $\{0,2\}$ & $\{1,2\}$ \\
\hline$\{0\}$ & $\{0,1\}$ & $\{0,2\}$ & $\{1,2\}$ \\
\hline$\{1\}$ & $\{1,2\}$ & $\{0,1\}$ & $\{0,2\}$ \\
\hline$\{2\}$ & $\{0,2\}$ & $\{1,2\}$ & $\{0,1\}$ \\
\hline
\end{tabular}

\begin{tabular}{|c|c|c|c|}
\hline$;$ & $\{0,1\}$ & $\{0,2\}$ & $\{1,2\}$ \\
\hline$\{0\}$ & $\{0,1\}$ & $\{0,2\}$ & $\{1,2\}$ \\
\hline$\{1\}$ & $\{0,2\}$ & $\{1,2\}$ & $\{0,1\}$ \\
\hline$\{2\}$ & $\{1,2\}$ & $\{0,1\}$ & $\{0,2\}$ \\
\hline
\end{tabular}

TABLE 9. Comparison of the differences in the relative multiplication tables for $\mathfrak{C}$ and $\mathfrak{A}_{9}$.

yields the same result as in $\mathfrak{C}$.

The Boolean axioms (R1)-(R3) obviously hold in $\mathfrak{A}_{9}$, because the Boolean part of $\mathfrak{A}_{9}$ coincides with the Boolean part of $\mathfrak{C}$. Similarly, the identity law (R5) and the first involution law (R6) hold trivially in $\mathfrak{A}_{9}$.

For the verification of the associative law (R4), observe that most instances of this axiom yield the same result in $\mathfrak{A}_{9}$ as in $\mathfrak{C}$. Since $\mathfrak{C}$ is a relation algebra, these instances must hold in $\mathfrak{C}$ and therefore also in $\mathfrak{A}_{9}$. This includes the following cases. (i) At least one of the elements $r, s$, and $t$ is empty; in this case, both sides of (R4) reduce to the empty set. (ii) All three elements are singletons of group elements, say

$$
r=\{f\}, \quad s=\{g\}, \quad t=\{h\} ;
$$

in this case, both sides of (R4) reduce to the singleton $\{f \circ g \circ h\}$. (iii) At least two of the three elements have cardinality at least two, and the third is not empty; in this case, both sides of (R4) reduce to the unit $\{0,1,2\}$. (iv) One of the elements is the unit, and the other two are non-empty; in this case, both sides of (R4) again reduce to the unit. (v) Both $s$ and $t$ are singletons; in this case, all relative products involved are computed the same way in $\mathfrak{A}_{9}$ as in $\mathfrak{C}$.

There remain two cases to consider. If $r$ and $s$ are singletons, and $t$ a doubleton, then

$$
\begin{aligned}
r ;(s ; t)=r^{-1} \circ\left(s^{-1} \circ t\right)=\left(r^{-1} \circ s^{-1}\right) \circ t & \\
= & (s \circ r)^{-1} \circ t=(r \circ s)^{-1} \circ t=(r ; s) ; t .
\end{aligned}
$$

The first and last equalities follow from the definition of ; and the assumption that $r$ and $s$ are singletons, and $t$ a doubleton. Notice in this connection that the relative product of two singletons is always a singleton, and the relative product of a singleton with a doubleton is always a doubleton, both in $\mathfrak{A}_{9}$ and in $\mathfrak{C}$. The second and third equalities follow from the validity of (R4) and (R7) in $\mathfrak{C}$. The fourth equality uses the fact that the operation of relative multiplication in $\mathfrak{C}$ is commutative (because the group underlying $\mathfrak{C}$ is commutative). If $r$ and $t$ are singletons, and $s$ a doubleton, then

$$
r ;(s ; t)=r^{-1} \circ(s \circ t)=\left(r^{-1} \circ s\right) \circ t=(r ; s) ; t,
$$

by the definition of relative multiplication in $\mathfrak{A}_{9}$, the assumptions on the three elements, and the validity of (R4) in $\mathfrak{C}$.

Turn now to the task of verifying the second involution law (R7) in $\mathfrak{A}_{9}$. As in the case of (R4), most instances of (R7) yield the same result in $\mathfrak{A}_{9}$ as in $\mathfrak{C}$, and are 
therefore automatically valid in $\mathfrak{A}_{9}$. This includes the case when at least one of the elements $r$ and $s$ is empty, in which case both sides of (R7) reduce to the empty set; the case when one of the elements is non-empty and the other is the unit $\{0,1,2\}$, in which case both sides of (R7) reduce to the unit; and the case when both $r$ and $s$ have at least two elements, in which case both sides of (R7) again reduce to the unit. There remain three cases to consider. If $r$ and $s$ are both singletons, then

$$
(r ; s)^{\smile}=r ; s=s ; r=s^{\smile} ; r^{\smile} .
$$

The first and last equalities use the fact that $r ; s$ is a singleton, and converse is the identity function on singletons in $\mathfrak{A}_{9}$. The second equality follows from the fact that relative multiplication in $\mathfrak{A}_{9}$ is commutative on singletons. If $r$ is a singleton, and $s$ a doubleton, then

$$
(r ; s)^{\smile}=\left(r^{-1} \circ s\right)^{\smile}=\left(r^{-1} \circ s\right)^{-1}=s^{-1} \circ\left(r^{-1}\right)^{-1}=s^{-1} \circ r=s^{\smile} ; r=s^{\smile} ; r^{\smile} .
$$

The first equality follows from the definition of relative multiplication in $\mathfrak{A}_{9}$, and the assumption that $r$ is a singleton and $s$ a doubleton. The second equality uses the fact that the operations of converse in $\mathfrak{C}$ and $\mathfrak{A}_{9}$ coincide on doubletons, and $r^{-1} \circ s$ must be a doubleton (since $r$ is a singleton and $s$ a doubleton). The third and fourth equalities use the validity of $(\mathrm{R} 7)$ and $(\mathrm{R} 6)$ in $\mathfrak{C}$. The fifth equality uses the fact that the operations of converse in $\mathfrak{C}$ and $\mathfrak{A}_{9}$ coincide on doubletons, and so do the operations of relative multiplication when the right-hand argument is a singleton. The sixth equality uses the fact that converse on singletons is the identity function in $\mathfrak{A}_{9}$, and $r$ is assumed to be a singleton. Finally, if $r$ is a doubleton, and $s$ a singleton, then

$$
(r ; s)^{\smile}=(r \circ s)^{-1}=s^{-1} \circ r^{-1}=s ; r^{\smile}=s^{\smile} ; r^{\smile} .
$$

The first and third equalities use the definitions of relative multiplication and converse in $\mathfrak{A}_{9}$, and the assumptions on $r$ and $s$; the second equality uses the validity of (R7) in $\mathfrak{C}$; and the last equality uses the fact that converse is the identity function on singletons in $\mathfrak{A}_{9}$.

Next, we verify the distributive law (R8) in $\mathfrak{A}_{9}$. As usual, most instances of this axiom yield the same result in $\mathfrak{A}_{9}$ as in $\mathfrak{C}$, and are therefore valid in $\mathfrak{A}_{9}$. This includes all cases when $t$ is not a doubleton. It also includes the case when $t$ is a doubleton and at least one of $r$ and $s$ has at least two elements (in which case, both sides of (R8) reduce to the unit, because in the relative product in $\mathfrak{A}_{9}$ and in $\mathfrak{C}$ of two elements with at least two elements is always the unit-see Table 21). The case when $t$ is a doubleton, and at least one of $r$ and $s$ is the empty set is trivial; for example, if $r$ is the empty set, then

$$
(r+s) ; t=s ; t \quad \text { and } \quad r ; t+s ; t=\varnothing ; t+s ; t=\varnothing+s ; t=s ; t .
$$

Similarly, the case when $r=s$ is trivial. There remains the case when $t$ is a doubleton, and $r$ and $s$ are distinct singletons. In this case, $r+s$ is a doubleton, so $(r+s) ; t$ is the unit. As $r ; t$ and $s ; t$ are distinct doubletons, the sum $r ; t+s ; t$ is also the unit. Thus, (R8) is valid in $\mathfrak{A}_{9}$ in this case as well.

It remains to verify Tarski's law (R10), or equivalently, (R10'), in $\mathfrak{A}_{9}$. The instances of (R10) in which $r$ is the empty set or has at least two elements yield the same result in $\mathfrak{A}_{9}$ as in $\mathfrak{C}$, and are therefore valid in $\mathfrak{A}_{9}$. The same is true of those instances of $\left(\underline{\mathrm{R} 10^{\prime}}\right)$ in which $r$ is a singleton, and $s$ is either the empty set or the unit. There remain two cases to consider. If $r$ and $s$ are both singletons, 
then $-(r ; s)$ in $\mathfrak{A}_{9}$ coincides with $-(r \circ s)$ in $\mathfrak{C}$ and is therefore a doubleton. It follows that

$$
r^{\smile} ;-(r ; s)=r ;-(r ; s)=r^{-1} \circ-(r \circ s) \leq-s .
$$

The first equality uses the fact that converse is the identity operation on singletons in $\mathfrak{A}_{9}$, the second uses the definition of relative multiplication in $\mathfrak{A}_{9}$ and the assumptions on $r$ and $s$, and the last uses the validity of ( 1 singleton, and $s$ a doubleton, then $r ; s$ is also a doubleton, so $-(r ; s)$ is a singleton. Consequently,

$$
r^{\smile} ;-(r ; s)=r ;-(r ; s)=r \circ-\left(r^{-1} \circ s\right)=\left(r^{-1}\right)^{-1} \circ-\left(r^{-1} \circ s\right) \leq-s .
$$

The first equality uses the fact that converse is the identity function on singletons in $\mathfrak{A}_{9}$, while the second equality uses the definition of relative multiplication in $\mathfrak{A}_{9}$, the assumptions on $r$ and $s$, and the observations preceding the calculation. The third equality uses the validity of $(\mathrm{R} 6)$ in $\mathfrak{C}$, and the final inequality follows from the validity of ( $\left.\mathrm{R} 10^{\prime}\right)$ in $\mathfrak{C}$ (with $r$ replaced by $r^{-1}$ ).

A computational check using Mace4 has shown that $\mathfrak{A}_{9}$ is the unique independence model for (R9) of cardinality 8 , and that there is no smaller independence model for this axiom.

It is interesting to note that the left-hand distributive law $(\underline{\mathrm{R} 8})$ for relative multiplication fails in $\mathfrak{A}_{9}$. Indeed, take $r, s$, and $t$ to be $\{1\},\{0\}$, and $\{2\}$ respectively to obtain

$$
r ;(s+t)=\{1\} ;(\{0\}+\{2\})=\{1\} ;\{0,2\}=\{1,2\},
$$

by the definition of ; (see Table 9), but

$$
r ; s+r ; t=\{1\} ;\{0\}+\{1\} ;\{2\}=\{1\} \circ\{0\}+\{1\} \circ\{2\}=\{1\}+\{0\}=\{0,1\} .
$$

\section{INDEPENDENCE OF (R10)}

Consider any Boolean algebra $(A,+,-)$ with at least two elements. Take relative multiplication to be the Boolean operation of addition, take converse to be the identity function on $A$, and take the identity element to be the Boolean zero. To see that (R10) fails in the resulting algebra $\mathfrak{A}_{10}$, take $r$ and $s$ to be 1 , and observe that

$$
r^{\smile} ;-(r ; s)=r+-(r+s)=1+-(1+1)=1 \not \leq 0=-s,
$$

by the definitions of relative multiplication and converse, and the choice of $r$ and $s$. On the other hand, the Boolean axioms (R1)-(R3) hold automatically in $\mathfrak{A}_{10}$, and (R4)-(R9) reduce to Boolean laws, so they, too, are valid in $\mathfrak{A}_{10}$. To give two concrete examples, consider (R7) and (R8). We have

$$
(r ; s)^{\smile}=r+s=s+r=s^{\smile} ; r^{\smile},
$$

and

$$
(r+s) ; t=(r+s)+t=(r+t)+(s+t)=r ; t+s ; t,
$$

for all elements $r, s$, and $t$, by the definition of relative multiplication and converse.

Start with a two-element Boolean algebra in the preceding construction to arrive at an independence model for (R10) of minimal cardinality.

There is another interesting and rather different independence model for (R10) that is worthwhile discussing. Start with the set relation algebra $\mathfrak{M}_{3}$ (see Table 1), 
and modify the operation of relative multiplication in two ways: require relative multiplication by the diversity element to always yield the diversity element,

$$
r ; 0^{\prime}=0^{\prime} ; r=0^{\prime}
$$

for all elements $r$, and require

$$
0 ; 1=1 ; 0=0
$$

(see Table 10).

\begin{tabular}{c|c|c|c|c|}
$;$ & 0 & $1^{\prime}$ & 0 & 1 \\
\hline 0 & 0 & 0 & 0 & 0 \\
\hline $1^{\prime}$ & 0 & $1^{\prime}$ & 0 & 1 \\
\hline $0^{\prime}$ & 0 & 0 & 0 & 0 \\
\hline 1 & 0 & 1 & 0 & 1 \\
\hline
\end{tabular}

TABLE 10. Relative multiplication table for $\mathfrak{B}_{10}$.

To see that (R10), or equivalently, ( $\left(\mathrm{R} 10^{\prime}\right)$, fails in the resulting algebra $\mathfrak{B}_{10}$, take $r$ and $s$ to be 0 ' to obtain

$$
r^{\smile} ;-(r ; s)=0^{\prime} ;-\left(0^{\prime} ; 0^{\prime}\right)=0^{\prime} ;-0^{\prime}=0^{\prime} ; 1^{\prime}=0^{\prime} \not \leq 1^{\prime}=-s,
$$

by the definition of relative multiplication and converse, and the choice of $r$ and $s$.

The Boolean part of $\mathfrak{B}_{10}$ coincides with the Boolean part of $\mathfrak{M}_{3}$, the operation of relative multiplication is commutative, 1' remains the identity element for relative multiplication, and converse is the identity function, so (R1)-(R3), (R5), (R6), (R7), and (R9) are all easily seen to be valid in $\mathfrak{B}_{10}$. It remains to verify (R4) and (R8). The relative multiplication table for $\mathfrak{B}_{10}$ differs from that of $\mathfrak{M}_{3}$ (compare Tables 1 and 100) in the seven entries

$$
0 ; 0^{\prime}, \quad 0^{\prime} ; 0, \quad 0 ; 1, \quad 1 ; 0, \quad 0^{\prime} ; 0^{\prime}, \quad 0^{\prime} ; 1, \quad \text { and } 1 ; 0^{\prime} \text {, }
$$

which all have the value 0 ' in $\mathfrak{B}_{10}$. Consequently, every instance of (R4) and (R8) which does not involve the computation of one of these products is automatically valid in $\mathfrak{B}_{10}$, because it is valid in $\mathfrak{M}_{3}$. Notice also that the relative product of two elements in $\mathfrak{B}_{10}$ is never 0 ' unless one of the elements is 0 ', or else one of the elements is 0 and the other is 1 (see Table 10). Consequently, if $r$ and $s$ are both different from 1', then $r$; $s$ is different from 0 ' if and only if $r$ and $s$ are either both 0 or both 1 .

The validity in $\mathfrak{B}_{10}$ of the associative law (R4) follows readily from the preceding observations. Any instance of (R4) in which at least one of the three elements $r, s$, and $t$ is 1 ' holds trivially in $\mathfrak{B}_{10}$, because both sides of $(\mathrm{R} 4)$ reduce to the relative product of the other two elements. Assume now that none of these three elements is 1'. In this case, none of the relative products involved in (R4) can have the value 1' (see Table 10), so the left side of (R4) is different from 0' if and only if $r$ and $s ; t$ are either both 0 or both 1 , by the observations at the end of the preceding paragraph. In the case under consideration, $s ; t$ can only be 0 or 1 if $s$ and $t$ are both 0 or both 1 respectively (see Table 10). Consequently, the left side of (R4) is different from 0 ' if and only if $r, s$, and $t$ are all 0 or all 1 . A similar remark applies to the right side of (R4). Thus, either both sides of (R4) evaluate to 0 ', in 
which case (R4) holds in $\mathfrak{B}_{10}$, or else $r, s$, and $t$ all have the same value either 0 or 1 - and in this case (R4) holds in $\mathfrak{B}_{10}$, because it holds in $\mathfrak{M}_{3}$.

Turn finally to the verification of $(\mathrm{R} 8)$ in $\mathfrak{B}_{10}$. It is to be shown that both sides of this axiom evaluate to the same element in $\mathfrak{B}_{10}$. If $t$ is $0^{\prime}$, then both sides evaluate to $0^{\prime}$, and if $t$ is $1^{\prime}$, then both sides evaluate to $r+s$. Consider next the case when $t$ is 0 . If at least one of $r$ and $s$ is 0 ' or 1, then both sides of (R8) evaluate to 0'. For example, if $r$ is $0^{\prime}$, then

$$
(r+s) ; t=\left(0^{\prime}+s\right) ; 0=0^{\prime} \text { and } r ; t+s ; t=0^{\prime} ; 0+s ; 0=0^{\prime},
$$

since $s ; 0$ is at any rate below 0 '. A similar argument applies when $r$ is 1 . The only other possibility in the case under consideration is that $r$ and $s$ both assume values in the set $\left\{0,1^{\prime}\right\}$, and in this case the computation of each side of (R8) yields the same result in $\mathfrak{B}_{10}$ as it does in $\mathfrak{M}_{3}$.

There remains the case when $t$ is 1 . Keep in mind that $0 ; 1$ and $0^{\prime} ; 1$ are both 0 ', and 1 '; 1 and $1 ; 1$ are both 1 (see Table 10). The sum $r+s$ assumes one of four values: $0,1^{\prime}, 0$ ', or 1 . If this value is $1^{\prime}$, then at least one of $r$ and $s$ must be 1' (since 1 ' is an atom), so both sides of (R8) evaluate to 1 . If the value of the sum is 1, then at least one of $r$ and $s$ is either 1' or 1, so both sides of (R8) again evaluate to 1 . If the value of the sum is 0 or 0 ', then neither $r$ nor $s$ can be 1' or 1 , and therefore both sides of (R8) must evaluate to 0 '. This completes the verification of $(\mathrm{R} 8)$ in $\mathfrak{B}_{10}$.

\section{A VARIANT OF TARSKI'S AXIOM SYSTEM}

Somewhat surprisingly, it turns out that by modifying slightly one of the axioms in Tarski's system, namely (R8), another of the axioms, namely (R7), becomes redundant. We begin with some lemmas that will be needed again later.

Lemma 1. Under the assumption of (R1)-(R3), axiom (R8) implies the left-hand monotony law for relative multiplication, and axiom $(\underline{\mathrm{R} 8}$ ) implies the right-hand hand monotony law for relative multiplication.

Proof. If $r \leq s$, then $s=r+s$, by the definition of $\leq$, and therefore

$$
s ; t=(r+s) ; t=r ; t+s ; t,
$$

by (R8). Consequently, $r ; t \leq s ; t$, by the definition of $\leq$. This proves that (R8) implies the left-hand monotony law for relative multiplication. A similar argument shows that $\left(\underline{\mathrm{R} 8^{\prime}}\right)$ implies the right-hand monotony law for relative multiplication.

Lemma 2. Under the assumption of (R1)-(R3), (R6), and $(\underline{\mathrm{R} 8})$, axiom (R10) is equivalent to the law

$$
(r ; s) \cdot t=0 \quad \text { if and only if } \quad\left(r^{\smile} ; t\right) \cdot s=0 .
$$

Proof. As was mentioned in Section 2, on the basis of (R1)-(R3), axiom (R10) is equivalent to $\left(\underline{\mathrm{R} 10^{\prime}}\right)$, so it suffices to prove that $\left(\underline{\mathrm{R} 10^{\prime}}\right)$ is equivalent to $\left(\mathrm{R} 11^{\prime}\right)$. Assume first that (R10') holds. If $(r ; s) \cdot t=0$, then $t \leq-(r ; s)$, by Boolean algebra (here we are using (R1)-(R3)), and therefore

$$
r^{\smile} ; t \leq r^{\smile} ;-(r ; s) \leq-s,
$$

by the right-hand monotony law for relative multiplication (which is valid under the assumption of $\left(\underline{\mathrm{R} 8^{\prime}}\right)$, by Lemma 1) and (R10). Consequently, $\left(r^{\smile} ; t\right) \cdot s=0$, 
by Boolean algebra. This argument establishes the implication from left to right in (R11').

To establish the reverse implication, assume $\left(r^{\smile} ; t\right) \cdot s=0$, and use the results of the previous paragraph (with $r^{\smile}, t$, and $s$ in place of $r, s$, and $t$ respectively) to obtain $\left(r^{\smile} ; s\right) \cdot t=0$. Apply $(\mathrm{R} 6)$ to conclude that $(r ; s) \cdot t=0$.

Assume now that $\left(\underline{\mathrm{R} 11^{\prime}}\right)$ holds. Take $t$ to be $-(r ; s)$ and observe that the left side of ( $111^{\prime}$ obviously holds, by Boolean algebra. Consequently, the right side must hold, that is to say,

$$
\left[r^{\smile} ;-(r ; s)\right] \cdot s=0 .
$$

This equation is clearly equivalent to $\left(\underline{\mathrm{R} 10^{\prime}}\right)$, by Boolean algebra.

As is clear from the proof of Lemma 2, under the assumption of (R1)-(R3) and $(\underline{\mathrm{R} 8}$ ), axiom (R10) is equivalent to the implication from left to right in (R11'), that is to say, (R10) is equivalent to (R11) (see Section 2).

Lemma 3. Under the assumption of (R6), (R7) and (R9), axiom (R8) is equivalent to $\left(\mathrm{R} 8^{\prime}\right)$.

Proof. The derivation of $\left(\mathrm{R} 8^{\prime}\right)$ from (R8) is contained in the proof of Theorem 1.21 in Chin-Tarski 1]. For the convenience of the reader, here are the details of the argument. Observe that

$$
\left[\left(r^{\smile}+s^{\smile}\right) ; t^{\smile}\right]^{\smile}=t^{\smile \smile} ;\left(r^{\smile}+s^{\smile}\right)^{\smile}=t^{\smile \smile} ;\left(r^{\smile \smile}+s^{\smile \smile}\right)=t ;(r+s),
$$

by (R7), (R9), and (R6), and

$$
\begin{aligned}
& {\left[\left(r^{\smile} ; t^{\smile}\right)+\left(s^{\smile} ; t^{\smile}\right)\right]^{\smile}=\left(r^{\smile} ; t^{\smile}\right)^{\smile}+\left(s^{\smile} ; t^{\smile}\right)^{\smile}}
\end{aligned}
$$

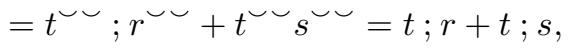

by (R9), (R7), and (R6). Axiom (R8) (with $r, s$, and $t$ replaced by $r^{\smile}, s^{\smile}$, and $t^{\smile}$ respectively) ensures that

$$
\left(r^{\smile}+s^{\smile}\right) ; t^{\smile}=r^{\smile} ; t^{\smile}+s^{\smile} ; t^{\smile} .
$$

Form the converse of both sides of this last equation, and use (10) and (2) to arrive at $(\underline{\mathrm{R} 8})$.

A dual argument leads to an analogous derivation of (R8) from $\left(\underline{R} 8^{\prime}\right)$.

The next lemma occurs as part of Theorem 313 in [18. We prove it here for the convenience of the reader.

Lemma 4. Under the assumption of (R1)-(R3), axioms (R4) and (R5), together with (R11'), imply (R7).

Proof. The key step in the argument is the proof of the equivalence

$$
(r ; s)^{\smile} \cdot t=0 \quad \text { if and only if } \quad\left(s^{\smile} ; r^{\smile}\right) \cdot t=0 .
$$


for all elements $r, s$, and $t$. To establish (1), observe that

$$
\begin{array}{rll}
(r ; s)^{\smile} \cdot t=0 \quad \text { if and only if } & {\left[(r ; s)^{\smile} ; 1^{\prime}\right] \cdot t=0,} \\
& \text { if and only if } & {[(r ; s) ; t] \cdot 1^{\prime}=0,} \\
\text { if and only if } & {[r ;(s ; t)] \cdot 1^{\prime}=0,} \\
\text { if and only if } & \left(r^{\smile} ; 1^{\prime}\right) \cdot(s ; t)=0, \\
\text { if and only if } & r^{\smile} \cdot(s ; t)=0, \\
\text { if and only if } & (s ; t) \cdot r^{\smile}=0, \\
\text { if and only if } & \left(s^{\smile} ; r^{\smile}\right) \cdot t=0 .
\end{array}
$$

The first equivalence uses (R5), the second uses (R11') (with $r ; s, t$, and $1^{\prime}$ in place of $r, s$, and $t$ respectively), the third uses (R4), the fourth uses (R11') (with $s ; t$ and 1 ' in place of $s$ and $t$ respectively), the fifth uses (R5), the sixth uses Boolean algebra, and the seventh uses (R11') (with $s, t$, and $r^{-}$in place of $r, s$ and $t$ respectively).

Turn now to the proof of the second involution law. Obviously,

$$
(r ; s)^{\smile} \cdot-\left[(r ; s)^{\smile]}=0,\right.
$$

by Boolean algebra, so

$$
\left(s^{\smile} ; r^{\smile}\right) \cdot-\left[(r ; s)^{\smile}\right]=0,
$$

by (11) (with $-\left[(r ; s)^{\smile}\right]$ in place of $t$ ). It follows by Boolean algebra that

$$
s^{\smile} ; r^{\smile} \leq(r ; s)^{\smile} \text {. }
$$

Similarly, it is obvious that

$$
\left(s^{\smile} ; r^{\smile}\right) \cdot-\left(s^{\smile} ; r^{\smile}\right)=0,
$$

by Boolean algebra. so

$$
(r ; s)^{\smile} \cdot-\left(s^{\smile} ; r^{\smile}\right)=0,
$$

by (11) (with $-\left(s^{\smile} ; r^{\smile}\right)$ in place of $t$ ). It follows by Boolean algebra that

$$
(r ; s)^{\smile} \leq s^{\smile} ; r^{\smile} .
$$

Combine (2) and (3) to arrive at the second involution law.

Take $\mathcal{R}$ to be the system of equations obtained from (R1)-(R10) by dropping (R7), and replacing the right-hand distributive law (R8) with its left-hand version ( $\underline{\mathrm{R} 8}$ ).

Theorem 2. The system of axioms $\mathcal{R}$ is equivalent to Tarski's system (R1)-(R10).

Proof. It is easy to check that Tarski's axioms imply the axioms in $\mathcal{R}$. In fact, it is only necessary to derive ( $\left(\underline{\mathrm{R}^{\prime}}\right)$ from (R1)-(R10), and this is done in Lemma 3 ,

To prove that, conversely, the axioms in $\mathcal{R}$ imply Tarski's axioms, it must be shown that (R7) and (R8) are derivable from $\mathcal{R}$. Apply Lemma 2 to obtain (R11'), and apply Lemma 4 to obtain (R7). An application of Lemma 3 now yields (R8). 


\section{The IndePendence of AXIOM System $\mathcal{R}$}

Interestingly, the axioms in $\mathcal{R}$ are also all independent of one another. For example, the left-hand distributive law $\left(\mathrm{R} 8^{\prime}\right.$ fails in the model $\mathfrak{A}_{8}$, while the remaining axioms of $\mathcal{R}$ are valid in $\mathfrak{A}_{8}$, so $\left(\underline{\mathrm{R} 8^{\prime}}\right.$ is independent of the other axioms of $\mathcal{R}$. In fact, the same assignment of values to $r, s$, and $t$ that invalidates (R8) in $\mathfrak{A}_{8}$ also invalidates $\left(\mathrm{R} 8^{\prime}\right)$, since relative multiplication and addition are commutative operations in $\mathfrak{A}_{8}$. Alternatively, $\mathrm{R} 8$ must fail in the independence model $\mathfrak{A}_{7}$, in which the remaining axioms of $\mathcal{R}$ are valid. Indeed, if $\left(\underline{\mathrm{R} 8^{\prime}}\right.$ were valid in $\mathfrak{A}_{7}$, then $\mathfrak{A}_{7}$ would be a model of $\mathcal{R}$, and therefore also of (R7), by Theorem 2 ; but we have seen that this is not the case. To obtain a concrete instance in which (R8) fails, let $r$ be any non-zero element in $\mathfrak{A}_{7}$, and let $s$ and $t$ be 1 ' and 0 ' respectively. The definition of relative multiplication in $\mathfrak{A}_{7}$ implies that

$$
r ;(s+t)=r ;\left(1^{\prime}+0 '\right)=r ; 1=0
$$

but

$$
r ; s+r ; t=r ; 1^{\prime}+r ; 0^{\prime}=r+0=r \neq 0 .
$$

This argument actually shows more than is claimed. Since (R8) is valid in $\mathfrak{A}_{7}$, axiom $\left(\mathrm{R} 8^{\prime}\right)$ is independent of the set of axioms (R1)-(R6), (R8), (R9), and (R10). We will need this observation later.

As regards the independence of $(\mathrm{R} n)$ in $\mathcal{R}$ for $1 \leq n \leq 6$ and $n=10$, the left-hand distributive law $\left(\overline{\mathrm{R} 8^{\prime}}\right)$ is valid in the independence model $\mathfrak{A}_{n}$ constructed above, so $\mathfrak{A}_{n}$ also serves to establish the independence of $(\mathrm{R} n)$ with respect to $\mathcal{R}$. However, the left-hand distributive law fails in $\mathfrak{A}_{9}$, so a new model must be constructed in order to establish the independence of (R9) with respect to $\mathcal{R}$.

The independence model $\mathfrak{B}_{9}$ for (R9) with respect to $\mathcal{R}$ is obtained from the relation algebra $\mathfrak{D}$ constructed in Table 5 by modifying the definitions of relative multiplication and converse. In order to avoid confusion of notation, write ; and $\smile$ for the operations of relative multiplication and converse to be defined in $\mathfrak{B}_{9}$, and write $\circ$ for the operation of relative multiplication in $\mathfrak{D}$; a separate notation for the operation of converse in $\mathfrak{D}$ is unnecessary, since this operation is defined to be the identity function. In $\mathfrak{B}_{9}$, converse is defined to interchange the elements

$$
1^{\prime}+a \text { and } 1^{\prime}+b,
$$

and to map every other element to itself. (Notice the similarity in intuition with the model $\mathfrak{A}_{9}$.) As a result, (R9) fails in $\mathfrak{B}_{9}$. Indeed, if $r$ and $s$ are taken to be 1 ' and $a$ respectively, then

$$
(r+s)^{\smile}=\left(1^{\prime}+a\right)^{\smile}=1^{\prime}+b \neq 1^{\prime}+a=r^{\smile}+s^{\smile} .
$$

As in the case of the algebra $\mathfrak{A}_{9}$, the change in the definition of converse requires a corresponding compensatory change in the definition of relative multiplication. If $r$ is one of the elements $1^{\prime}+a$ and $1^{\prime}+b$, and $s$ is one of the atoms $a$ and $b$, then the relative product $r ; s$ in $\mathfrak{B}_{9}$ is defined by

$$
r ; s=r^{\smile \circ s .}
$$

In all other cases, relative multiplication in $\mathfrak{B}_{9}$ is defined to coincide with relative multiplication in $\mathfrak{D}$. Thus, only four entries in Table 5 are changed in the passage from $\mathfrak{D}$ to $\mathfrak{B}_{9}$ (see Table 11). Notice that the preceding equation is actually valid for all choices $r$ and $s$ except when $r$ is one of $1^{\prime}+a$ and $1^{\prime}+b$, and $s$ is 1 '. Indeed, 
if $r$ is different from $1^{\prime}+a$ and $1^{\prime}+b$, then $r ; s$ and $r^{\smile}$ coincide with $r \circ s$ and $r$ respectively, by definition, so that

$$
r ; s=r \circ s=r^{\smile} \circ s .
$$

On the other hand, if $r$ is one of $1^{\prime}+a$ and $1^{\prime}+b$, and $s$ is different from 1 ', then either $s$ is 0 , in which case the desired equality holds trivially; or $s$ is one of $a$ and $b$, in which case the equality holds by definition; or $s$ is the sum of at least two atoms, in which case both $r ; s$ and $r^{\smile} \circ s$ are equal to 1, yielding again the desired equality (see Table 5).

\begin{tabular}{|c|c|c|}
\hline$\circ$ & $a$ & $b$ \\
\hline $1^{\prime}+a$ & 1 & $0^{\prime}$ \\
\hline $1^{\prime}+b$ & $0 '$ & 1 \\
\hline
\end{tabular}

\begin{tabular}{|c|c|c|}
\hline$;$ & $a$ & $b$ \\
\hline $1^{\prime}+a$ & 0 & 1 \\
\hline $1^{\prime}+b$ & 1 & 0 \\
\hline
\end{tabular}

TABLE 11. Comparison of the differences in the relative multiplication tables for $\mathfrak{D}$ and $\mathfrak{B}_{9}$.

Axioms (R1)-(R3), (R5), and (R6) obviously all hold in $\mathfrak{B}_{9}$, so it remains to verify the validity of (R4), (R7), $\left(\underline{\mathrm{R} 8^{\prime}}\right)$, and (R10). Consider first (R4). If one of the elements $r, s$, and $t$ is 0 , then both sides of (R4) reduce to 0 in $\mathfrak{B}_{9}$, and if one of these elements is 1', then both sides of (R4) reduce to the relative product of the other two elements. In all other cases, both sides of (R4) reduce to 1 in $\mathfrak{B}_{9}$. In more detail, the relative product of two elements different from 0 and 1' is either 0 ' or 1 , and the relative product of these last two elements with any element different from 0 and 1 ' is always 1 (see Tables 5 and 11).

Turn now to the verification of (R7). If $r$ or $s$ is 1 ', then both sides of (R7) reduce to $s^{\smile}$ or $r^{\smile}$ respectively, so in this case (R7) holds trivially. In all other cases, we have

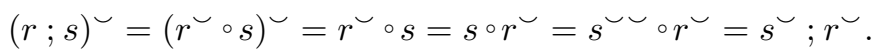

The first and last equalities follow from the definition of relative multiplication in $\mathfrak{B}_{9}$ and the assumption that neither $r$ nor $s$ is 1 ' (see the observations made above). The second equality uses the fact that $r^{\smile} \circ s$ is either $0,0^{\prime}$, or 1 in all cases under consideration, and converse maps each of these elements to itself in $\mathfrak{B}_{9}$. The third equality uses the fact that relative multiplication in $\mathfrak{D}$ is commutative, and the fourth uses the validity of $(\mathrm{R} 6)$ in $\mathfrak{B}_{9}$.

The next task is the verification of $\left(\overline{\mathrm{R} 8^{\prime}}\right)$. For all values of $r$ except $1^{\prime}+a$ and $1^{\prime}+b$, the computation of both sides of $\left(\underline{\mathbf{R} 8^{\prime}}\right)$ is the same in $\mathfrak{B}_{9}$ as it is in $\mathfrak{D}$, so these instances of $\left(\underline{\mathrm{R} 8^{\prime}}\right)$ are all valid in $\mathfrak{B}_{9}$. Also, if $t$ is 0 , or if $s=t$, then both sides of $\left(\overline{\mathrm{R} 8^{\prime}}\right)$ reduce to $r ; s$, and analogously if $s$ is 0 , so these instances of $\left(\overrightarrow{\mathrm{R} 8^{\prime}}\right)$ are also valid in $\mathfrak{B}_{9}$. Assume now that $r$ is one of $1^{\prime}+a$ and $1^{\prime}+b$, and that $s$ and $t$ are distinct non-zero elements. If neither $s$ nor $t$ is $1^{\prime}$, then

$$
r ;(s+t)=r^{\smile} \circ(s+t)=r^{\smile} \circ s+r^{\smile} \circ t=r ; s+r ; t,
$$

by the definition of relative multiplication in $\mathfrak{B}_{9}$ and the validity of $\left(\underline{R 8^{\prime}}\right)$ in $\mathfrak{D}$, so these instances of $\left(\vec{R} 8^{\prime}\right)$ hold in $\mathfrak{B}_{9}$. On the other hand, if $s$ is $1^{\prime}$, then $r ; s$ is $r$, which is above 1' by assumption; and $t$ is a non-zero element different from 1', by assumption; so $r$; $t$ must either be 0 ' or 1 , and therefore

$$
r ; s+r ; t \geq 1^{\prime}+0^{\prime}=1 .
$$


Since $s+t$ is the sum of at least two atoms,

$$
r ;(s+t)=1,
$$

by Table 5. and therefore all such instances of ( $\mathrm{R} 8^{\prime}$ hold in $\mathfrak{B}_{9}$ as well. A similar argument applies if $t$ is 1 '. This completes the verification of $\left(\sqrt{\mathrm{R} 8^{\prime}}\right)$ in $\mathfrak{B}_{9}$.

Turn finally to the verification of (R10), or equivalently, (R10' . If $r$ is different from both 1' $+a$ and 1' $+b$, then the computation of

$$
r^{\smile} ;-(r ; s)
$$

is the same in $\mathfrak{B}_{9}$ as it is in $\mathfrak{D}$, and consequently (11) must be below $-s$, by the validity of $\left(\mathrm{R} 10^{\prime}\right)$ in $\mathfrak{D}$. Suppose now that $r$ is one of $1^{\prime}+a$ and $1^{\prime}+b$. If $s$ is 0 , then $-s$ is 1 , so obviously (1) is below $-s$. If $s$ is the sum of at least two atoms, then $r ; s$ is 1 , and therefore $-(r ; s)$ is 0 (see Table 5). Consequently, (1) reduces to 0, which is below $-s$. If $s$ is $1^{\prime}$, then (11) reduces to $r^{\smile} ;-r$, which in the cases under consideration must yield 0', by Table 11 and the definition of converse in $\mathfrak{B}_{9}$ (since in this case $-r$ is the subdiversity atom that is below $r^{\smile}$ ). Also, $-s$ is 0 ', so (1) is equal to $-s$. In the remaining cases, $s$ is one of $a$ and $b$. Consequently, $r ; s$ assumes one of two values 0 ' or 1 , according to whether $s$ is, or is not, the subdiversity atom below $r$ (see Table 11). In the first case, $-(r ; s)$ is $1^{\prime}$, so (11) reduces to $r^{\smile}$, which coincides with $-s$, by Table 11 and the definition of relative multiplication. For example, if $r$ is $1^{\prime}+a$ and $s$ is $a$, then

$$
\begin{aligned}
& r^{\smile} ;-(r ; s)=\left(1^{\prime}+a\right)^{\smile} ;-\left(\left(1^{\prime}+a\right) ; a\right)=\left(1^{\prime}+a\right)^{\smile} ;-0^{\prime} \\
& =\left(1^{\prime}+a\right)^{\smile} ; 1^{\prime}=\left(1^{\prime}+a\right)^{\smile}=\left(1^{\prime}+b\right)=-s .
\end{aligned}
$$

In the second case, $r ; s$ is 1 , by Table 11, so $-(r ; s)$ is 0 , and therefore (1) reduces to 0 , which is certainly below $-s$. This completes the verification of ( $\left.\mathrm{R}^{\prime \prime}\right)$.

The following theorem has been proved.

Theorem 3. The set of axioms $\mathcal{R}$ is independent.

Notice that (R8) fails in $\mathfrak{B}_{9}$. For instance, if $r, s$, and $t$ are 1 ', $a$, and $b$ respectively, then

$$
(r+s) ; t=\left(1^{\prime}+a\right) ; b=1 \quad \text { and } \quad r ; t+s ; t=1^{\prime} ; b+a ; b=b+0^{\prime}=0 \text { '. }
$$

A computational check using Mace4 has shown that there is no independence model for (R9) of cardinality less than 8 , so $\mathfrak{B}_{9}$ is a minimal independence model for this axiom with respect to the axiom system $\mathcal{R}$.

\section{A SECOND VARIANT OF TARSKI's AXIOM SYSTEM}

As was pointed out above, in the independence models $\mathfrak{A}_{9}$ and $\mathfrak{B}_{9}$ for $(\mathrm{R} 9)$, the right-hand and left-hand distributive laws for relative multiplication fail respectively. This raises the question of whether (R9) is derivable from (R1)-(R8), (R8'), and (R10). As it turns out, (R9) is so derivable, and in fact even more is true: if $\left(\mathrm{R} 8^{\prime}\right)$ is added to Tarski's original axiom system, then both (R7) and (R9) become redundant in the sense that they are both derivable from the remaining axioms of the system.

Lemma 5. Under the assumption of (R1)-(R3), axioms (R5) and (R8), together with (R11'), imply (R9). 
Proof. The proof is very similar to the proof of Lemma 4. The key step in the argument is the proof of the equivalence

$$
(r+s)^{\smile} \cdot t=0 \quad \text { if and only if } \quad\left(r^{\smile}+s^{\smile}\right) \cdot t=0 .
$$

for all elements $r, s$, and $t$. To establish (1), observe that

$$
\begin{array}{rll}
(r+s)^{\smile} \cdot t=0 \quad \text { if and only if } & {\left[(r+s)^{\smile} ; 1^{\prime}\right] \cdot t=0,} \\
\text { if and only if } & {[(r+s) ; t] \cdot 1^{\prime}=0,} \\
\text { if and only if } & {[(r ; t)+(s ; t)] \cdot 1^{\prime}=0,} \\
\text { if and only if } & (r ; t) \cdot 1^{\prime}+(s ; t) \cdot 1^{\prime}=0, \\
\text { if and only if } & \left(r^{\smile} ; 1^{\prime}\right) \cdot t+\left(s^{\smile} ; 1^{\prime}\right) \cdot t=0, \\
\text { if and only if } & r^{\smile} \cdot t+s^{\smile} \cdot t=0, \\
\text { if and only if } & \left(r^{\smile}+s^{\smile}\right) \cdot t=0 .
\end{array}
$$

The first and sixth equivalences use (R5), the second uses (R11') (with $r+s, t$, and 1 ' in place of $r, s$, and $t$ respectively), the third uses (R8), the fourth and seventh use Boolean algebra, and the fifth uses (R11') twice (the first time with $t$ and $1^{\prime}$ in place of $s$ and $t$ respectively, and the second time with $s, t$ and 1 ' in place of $r, s$ and $t$ respectively).

Turn now to the proof of the second involution law. Obviously,

$$
(r+s)^{\smile} \cdot-\left[(r+s)^{\smile}\right]=0
$$

by Boolean algebra, so

$$
\left(r^{\smile}+s^{\smile}\right) \cdot-\left[(r+s)^{\smile}\right]=0,
$$

by (11) (with $-\left[(r+s)^{\smile}\right]$ in place of $t$ ). It follows by Boolean algebra that

$$
r^{\smile}+s^{\smile} \leq(r+s)^{\smile} \text {. }
$$

Similarly, it is obvious that

$$
\left(r^{\smile}+s^{\smile}\right) \cdot-\left(r^{\smile}+s^{\smile}\right)=0,
$$

by Boolean algebra, so

$$
(r+s)^{\smile} \cdot-\left(r^{\smile}+s^{\smile}\right)=0,
$$

by (11) (with $-\left(r^{\smile}+s^{\smile}\right.$ ) in place of $t$ ). It follows by Boolean algebra that

$$
(r+s)^{\smile} \leq r^{\smile}+s^{\smile} \text {. }
$$

Combine (2) and (3) to arrive at (R9).

Take $\mathcal{S}$ to be the axiom system consisting of equations (R1)-(R6), (R8), ( $(\mathrm{R} 8$ '), and (R10).

Theorem 4. The system of axioms $\mathcal{S}$ is equivalent to Tarski's system (R1)-(R10).

Proof. It is easy to check that Tarski's axioms imply the axioms in $\mathcal{S}$. In fact, it is only necessary to derive $\left(\underline{\mathrm{R} 8^{\prime}}\right)$ from (R1)-(R10), and that is done in Lemma 3 
To prove that, conversely, the axioms in $\mathcal{S}$ imply Tarski's axioms, it must be shown that $(\mathrm{R} 7)$ and $(\mathrm{R} 9)$ are derivable from $\mathcal{S}$. Observe first that $\left(\mathrm{R} 11^{\prime}\right)$ is derivable from $\mathcal{S}$, by Lemma 2 . Consequently, $(\mathrm{R} 7)$ is derivable from $\mathcal{S}$, by Lemma 4 . and (R9) is derivable from $\mathcal{S}$, by Lemma 5

\section{The INDEPENDENCE OF AXIOM SYSTEM $\mathcal{S}$}

The axioms in $\mathcal{S}$ are independent of one another. Indeed, as was already pointed out in the first paragraph of Section 16, the left-hand distributive law ( $\left.\mathrm{R} 8^{\prime}\right)$ fails in the model $\mathfrak{A}_{7}$, while the remaining axioms of $\mathcal{S}$ are valid in $\mathfrak{A}_{7}$. Consequently, (R8' is independent of the other axioms of $\mathcal{S}$. Similarly, it was proved in Section 16 that (R8) fails in the model $\mathfrak{B}_{9}$, while (R1)-(R6), (R8), and (R10) all hold in that model. Consequently, (R8) is independent of the remaining axioms in $\mathcal{S}$. As regards the independence of axioms $(\mathrm{R} n)$ in $\mathcal{S}$ for $1 \leq n \leq 6$ and $n=10$, axiom ( $\left(\mathrm{R} 8^{\prime}\right)$ is valid in the independence model $\mathfrak{A}_{n}$, so $\mathfrak{A}_{n}$ also serves to establish the independence of $(\mathrm{R} n)$ in $\mathcal{S}$.

Theorem 5. The set of axioms $\mathcal{S}$ is independent.

\section{REFERENCES}

[1] Chin, L. H. and Tarski, A.: Distributive and modular laws in the arithmetic of relation algebras. University of California Publications in Mathematics, New Series 1 (1951), pp. 341384.

[2] De Morgan, A.: On the syllogism, no. IV, and on the logic of relations. Transactions of the Cambridge Philosophical Society 10 (1864), pp. 331-358.

[3] Henkin, L., Monk, J. D., and Tarski, A.: Cylindric Algebras, Part I. Studies in Logic and the Foundations of Mathematics, vol. 64, North-Holland, Amsterdam, 1971, vi + 508 pp.

[4] Hirsch, R. and Hodkinson, I.: Relation algebras by games. Studies in Logic and the Foundations of Mathematics, vol. 147, Elsevier Science, North-Holland, Amsterdam, 2002, 712 pp.

[5] Jónsson, B.: Varieties of relation algebras, Algebra Universalis 15 (1982), pp. 273-298.

[6] Jónsson, B.: The theory of binary relations. In: H. Andréka, J. D. Monk, and I. Németi (eds.), Algebraic Logic, Colloquia Mathematica Societatis János Bolyai, vol. 54, NorthHolland Publishing Company, Amsterdam, 1991, pp. 245-292.

[7] Jónsson, B. and Tarski, A.: Representation problems for relation algebras. Bulletin of the American Mathematical Society 54 (1948), pp. 80 and 1192, Abstract 89.

[8] Jónsson, B. and Tarski, A.: Boolean algebras with operators, Part II. American Journal of Mathematics 74 (1952), pp. 127-162.

[9] Kamel, H.: Relational algebra. Doctoral Dissertation, University of Pennsylvania, Philadelphia PA, 1952, viii + 161 pp.

[10] Kamel, H.: Relational algebra and uniform spaces. Journal of the London Mathematical Society (1954), pp. 342-344.

[11] Löwenheim, L.: Über Möglichkeiten im Relativkalkül. Mathematische Annalen 76 (1915), pp. 447-470.

[12] Lyndon, R. C.: The representation of relational algebras, II. Annals of Mathematics, series 2,63 (1956), pp. 294-307.

[13] Maddux, R. D.: Topics in relation algebras. Doctoral dissertation, University of California at Berkeley, 1978, iii+ 241 pp.

[14] Maddux, R. D.: Some varieties containing relation algebras. Transactions of the American Mathematical Society 272 (1982), pp. 501-526.

[15] Maddux, R. D.: A sequent calculus for relation algebras. Annals of Pure and Applied Logic 25 (1983), pp. 73-101.

[16] Maddux, R. D.: Necessary subalgebras of simple nonintegral semiassociative relation algebras. Algebra Universalis 27 (1990), pp. 544-558.

[17] Maddux, R. D.: Pair-dense relation algebras. Transactions of the American Mathematical Society 328 (1991), pp. 83-131. 
[18] Maddux, R. D.: Relation algebras. Studies in Logic and the Foundations of Mathematics, vol. 150, Elsevier Science, North-Holland, Amsterdam, 2006, xxvi + 731 pp.

[19] McCune, W.: Prover9 and Mace4, http://www.cs.unm.edu/ ${ }^{\sim}$ mccune/mace4, 2005-2010.

[20] McKenzie, R. N.: Representations of integral relation algebras. Michigan Mathematical Journal 17 (1970), pp. 279-287.

[21] Monk, J.D.: On representable relation algebras, Michigan Mathematical Journal 11 (1964), pp. 207-210.

[22] Peirce, C.S.: Note B. The logic of relatives. In: C.S. Peirce (ed.) Studies in logic by members of the Johns Hopkins University, Little, Brown, and Company, Boston, 1883, pp. 187-203. [Reprinted by John Benjamins Publishing Company, Amsterdam, 1983.]

[23] Russell, B.: The principles of mathematics. Cambridge University Press, 1903. [Reprinted by Allen \& Unwin, London, 1948.]

[24] Schröder, E.: Vorlesungen über die Algebra der Logik (exakte Logik), vol. III, Algebra und Logik der Relative, part 1. B. G. Teubner, Leipzig, 1895. [Reprinted by Chelsea Publishing Company, New York, 1966.]

[25] Tarski, A.: On the calculus of relations. Journal of Symbolic Logic 6 (1941), pp. 73-89.

[26] Tarski, A.: Book manuscript containing some of Tarski's early contributions to the theory of relation algebras, written during the period 1943 to 1945 . The book was never published, but most of the results in the book were later included in [27.

[27] Tarski, A. and Givant, S.: A formalization of set theory without variables. Colloquium Publications, vol. 41, American Mathematical Society, Providence RI, 1987, xxi +318 pp.

Alfréd Rényi Institute of Mathematics, Hungarian Academy of Sciences, Budapest, PF. 127, H-1364 Hungary.

E-mail address: andreka@renyi.hu

Mills College, Department of Mathematics and Computer Science, 5000 MacArthur Boulevard, OAKland, CA 94613, United States

E-mail address: givant@mills.edu

Chapman University, Faculty of Mathematics, School of Computational Sciences, 545 West Palm Avenue, Orange, CA 92866, United States

E-mail address: jipsen@chapman.edu

Alfréd Rényi Institute of Mathematics, Hungarian Academy of Sciences, Budapest, PF. 127, H-1364 Hungary.

E-mail address: nemeti.istvan@mta.renyi.hu 